\title{
Novel regulatory role of neuropilin-1 in endothelial-to-mesenchymal transition and fibrosis in pancreatic ductal adenocarcinoma
}

\author{
Pratiek N. Matkar ${ }^{1,6}$, Krishna Kumar Singh 2,3,6, Dmitriy Rudenko ${ }^{1}$, Yu Jin Kim ${ }^{1}$, Michael \\ A. Kuliszewski ${ }^{1}$, Gerald J. Prud'homme ${ }^{4}$, David W. Hedley ${ }^{5}$, Howard Leong-Poi ${ }^{1,6}$ \\ ${ }^{1}$ Division of Cardiology, Keenan Research Centre for Biomedical Science, St. Michael's Hospital, Toronto, Canada \\ ${ }^{2}$ Division of Vascular Surgery, Keenan Research Centre for Biomedical Science, St. Michael's Hospital, Toronto, Canada \\ ${ }^{3}$ Division of Cardiac Surgery, Keenan Research Centre for Biomedical Science, St. Michael's Hospital, Toronto, Canada \\ ${ }^{4}$ Division of Laboratory Medicine, Keenan Research Centre for Biomedical Science, St. Michael's Hospital, Toronto, Canada \\ ${ }^{5}$ Division of Medical Oncology and Hematology, Ontario Cancer Institute, Campbell Family Cancer Research Institute, Princess \\ Margaret Cancer Centre, Toronto, Canada \\ ${ }^{6}$ Institute of Medical Science, University of Toronto, Toronto, Canada
}

Correspondence to: Howard Leong-Poi, email: leong-poih@smh.ca Krishna Kumar Singh, email: singhk@smh.ca

Keywords: endothelial cell, neuropilin-1, endothelial-to-mesenchymal transition, fibrosis, pancreatic cancer

Received: January 21, $2016 \quad$ Accepted: July 18, $2016 \quad$ Published: August 11, 2016

\section{ABSTRACT}

Pancreatic ductal adenocarcinoma (PDAC) is characterized by an intense fibrotic reaction termed tumor desmoplasia, which is in part responsible for its aggressiveness. Endothelial cells have been shown to display cellular plasticity in the form of endothelialto-mesenchymal transition (EndMT) that serves as an important source of fibroblasts in pathological disorders, including cancer. Angiogenic co-receptor, neuropilin-1 (NRP-1) actively binds TGF $\beta 1$, the primary mediator of EndMT and is involved in oncogenic processes like epithelial-to-mesenchymal transition (EMT). NRP-1 and TGF $\beta 1$ signaling have been shown to be aberrantly up-regulated in PDAC. We report herein a positive correlation between NRP-1 levels, EndMT and fibrosis in human PDAC xenografts. Loss of NRP-1 in HUVECs limited TGF $\beta 1$-induced EndMT as demonstrated by gain of endothelial and loss of mesenchymal markers, while maintaining endothelial cell architecture. Knockdown of NRP-1 down-regulated TGF $\beta$ canonical signaling (pSMAD2) and associated pro-fibrotic genes. Overexpression of NRP-1 exacerbated TGF $\beta 1$-induced EndMT and up-regulated TGF $\beta$ signaling and expression of pro-fibrotic genes. In vivo, loss of NRP-1 attenuated tumor perfusion and size, accompanied by reduction in EndMT and fibrosis. This study defines a previously unrecognized role of NRP-1 in regulating TGF $\beta 1$-induced EndMT and fibrosis, and advocates NRP-1 as a therapeutic target to reduce tumor fibrosis and PDAC progression.

\section{INTRODUCTION}

Despite improvements in our understanding of cancer biology and advances in cancer therapies, malignant tumors remain a leading cause of morbidity and mortality [1-3]. Pancreatic adenocarcinoma (PDAC) is a leading cause of mortality worldwide, with the lowest five-year survival rate [1,4-6]. As a consequence, the advancement of novel therapeutic strategies that specifically target the underlying biological processes involved in pancreatic cancer growth and metastases remains important [7]. Given the critical role of angiogenesis in cancer biology, anti- angiogenic therapies have been in the forefront of cancer therapeutics for decades [8-11]. Less vascularized tumors like PDAC, however remain largely incurable despite the significant advances in anti-angiogenesis research $[12,13]$. Morphologically, PDAC is composed of epithelial cells with variable degrees of ductal differentiation, surrounded by an intense fibrotic reaction called tumor desmoplasia, often referred to as tumor stroma/fibrosis [14-18]. Cancerassociated fibroblasts (CAFs) along with other stromal cells constitute the majority of the PDAC tumor [19, 20]. Recent evidence has demonstrated that CAFs contribute to several cancer initiating and promoting events by altering 
the tumor microenvironment through the release of oncogenic and angiogenic factors [20-22]. Furthermore, the characteristically dense desmoplastic reaction found in PDAC, including the stromal cells, extracellular matrix components and high interstitial pressure within the PDAC tumors can impair drug delivery and collectively contribute towards chemoresistance [13, 23-25]. Recently approved therapies targeting desmoplasia/fibrosis [26-28] opens new avenues for discovering novel strategies to suppress desmoplasia.

Although the origin of fibroblasts is complex and dependent on several factors, the importance of endothelial-to-mesenchymal transition (EndMT) as an important source of activated fibroblasts is an emerging concept [29-31]. Besides the crucial function carried out as a part of the endothelium, endothelial cells have displayed the ability of acquiring an extreme form of cell plasticity, whereby they acquire a mesenchymal cell phenotype. Primarily, EndMT is characterized by the acquisition of mesenchymal cell surface markers such as $\mathrm{N}$-cadherin, $\alpha \mathrm{SMA}$ and types I/III collagen with the corresponding loss of endothelial cell surface markers like VE-cadherin, CD31, etc. [29, 31]. While tightly regulated, EndMT is critical in the development of the primitive heart [32] and wound healing [33], and maladaptive EndMT has been linked to a variety of fibrotic pathologies including cancer $[31,34]$. Zeisberg et al. demonstrated convincing genetic evidence for EndMT-derived CAFs in the tumor microenvironment, whereby up to $40 \%$ of CAFs were derived via EndMT [35]. Mechanistically, several studies have established the involvement of TGF $\beta$ signaling in modulating EndMT [36-39]. However, mechanistic work involving EndMT in the context of diseased states like cancer remains obscure and warrants further investigation.

Initially implicated in the development of the nervous system [40], growing evidence suggests a significant role for neuropilin-1 (NRP-1) in cancer where it appears to be involved in angiogenesis and other aspects of tumor progression [41-48]. NRP-1 has been identified as a co-receptor for multiple growth factors, including VEGF-A, FGF, HGF and others [44, 49-52], and is expressed on both endothelial and tumor cells $[53,54]$. Our group has previously demonstrated that NRP-1 is also a co-receptor for TGF $\beta 1$, where it could enhance responses to both the active and latent forms of this cytokine [51]. Emerging data support TGF $\beta 1$ as a major modulator of tumor progression through regulation of endothelial cell proliferation and migration, extracellular matrix metabolism, epithelial-to-mesenchymal transition (EMT) and metastasis [55-59]. Furthermore, PDAC is characterized by aberrant expression of NRP-1 $[45,60,61]$, often correlated with tumor progression and poor patient prognosis [62]. Interestingly, recent evidences have implicated a novel regulatory role of NRP-1 in epithelial-to-mesenchymal transition (EMT); an evolutionary conserved developmental process, which is evoked during tumor invasion and metastasis of several cancers $[63,64]$. Similarly, recent studies suggest that endothelial-to-mesenchymal transition (EndMT), which is originally observed during heart development, has a major role in pathological settings of cancer and fibrosis [21-25, 31]. Transforming growth factor $\beta$ (TGF $\beta$ ) plays pivotal roles in both EMT and EndMT $[65,66]$. Finally, the precise function of NRP-1 in TGF $\beta$-mediated EndMT becomes highly relevant, given the importance of the profibrotic cytokine in tumor progression [22, 58, 67, 68], and the involvement of NRP-1 in pancreatic cancer and EMT.

In the current study, we hypothesized that NRP- 1 is crucial in regulating TGF $\beta 1$-induced EndMT and associated fibrosis in PDAC. To test our hypothesis, we utilized translational loss of function and over-expression study approaches. Firstly, we demonstrate a robust positive correlation between NRP-1 expression, EndMT and associated fibrosis in human PDAC xenografts. Using loss of function and overexpression studies in vitro, we demonstrate a novel regulatory role of NRP- 1 in TGF $\beta 1$-induced EndMT and fibrosis. Knockdown of NRP-1 limited while NRP-1 overexpression exacerbated TGF $\beta 1$-induced EndMT respectively. Overall, these data reveal a previously undetermined role of NRP- 1 in regulating TGF $\beta 1$-induced EndMT as a potential therapeutic target to limit EndMT-associated fibrosis in PDAC.

\section{RESULTS}

\section{Human PDAC tissue shows positive correlation between NRP-1 expression, EndMT and fibrosis}

H\&E staining of human tumor tissues displayed a typical ductal morphology of PDAC (Figure 1A). Further characterization of the xenografts is summarized in Tables 1 and 2. NRP-1 was aberrantly expressed in all the tissue samples at varying levels as assessed by immunohistochemistry studies (Figure 1A). Furthermore, collagen content as detected by Masson's trichrome staining, demonstrated a significant positive correlation with NRP-1 expression (Figure 1A, 1B). We observed a consistent increase in the expression of EndMT markers and pro-fibrotic markers with increasing NRP-1 expression at the transcript (Figure 1C) and protein level (Figure 1D, 1E). Contrarily, low levels of EndMT and pro-fibrotic markers were associated with reduced NRP-1 expression at the transcript and protein level (Figure 1C, 1D, 1E). Overall, we observed a robust positive correlation between NRP-1 expression, EndMT markers and pro-fibrotic genes in human PDAC tissue (Figure 1F), suggesting a previously undetermined role of NRP-1 in regulating EndMT and associated fibrosis in pancreatic tumors. 
A

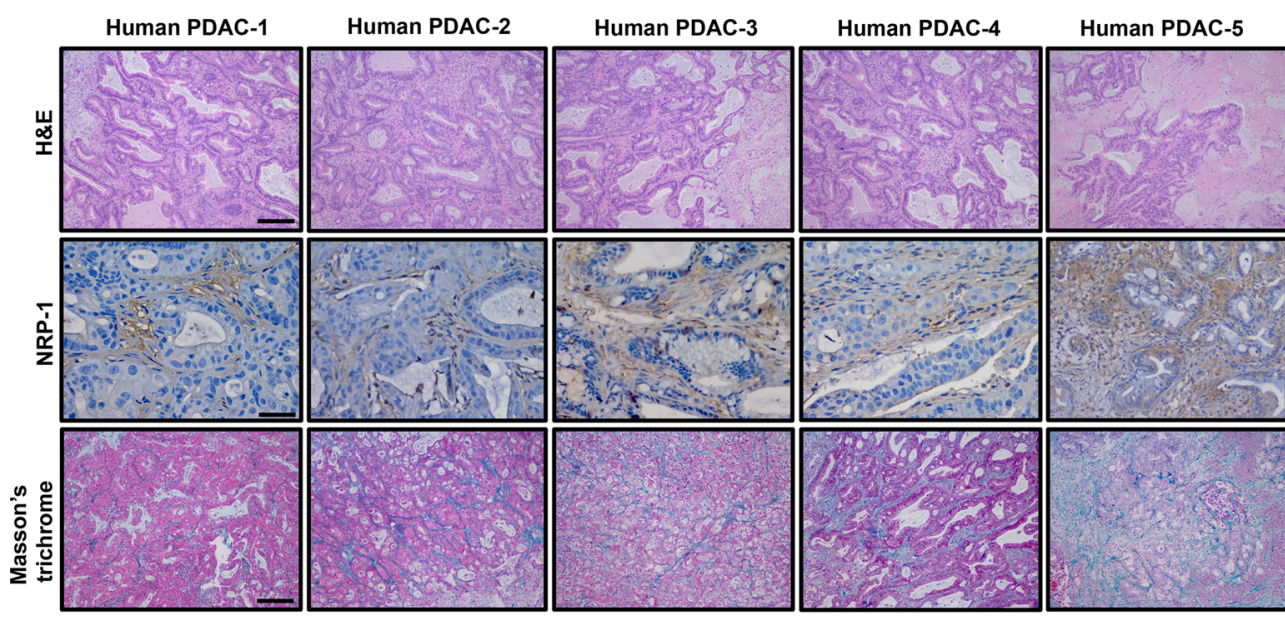

C
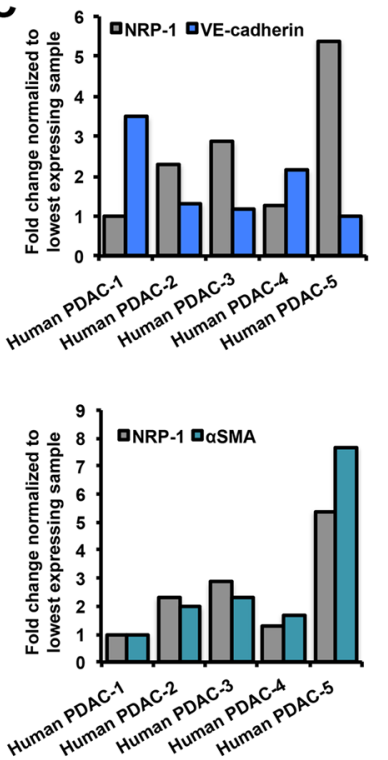
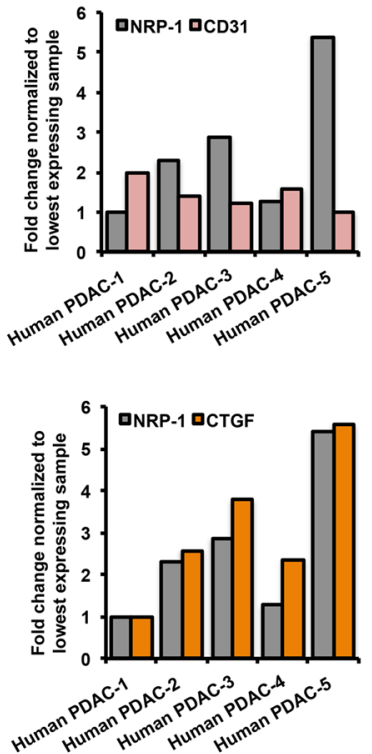
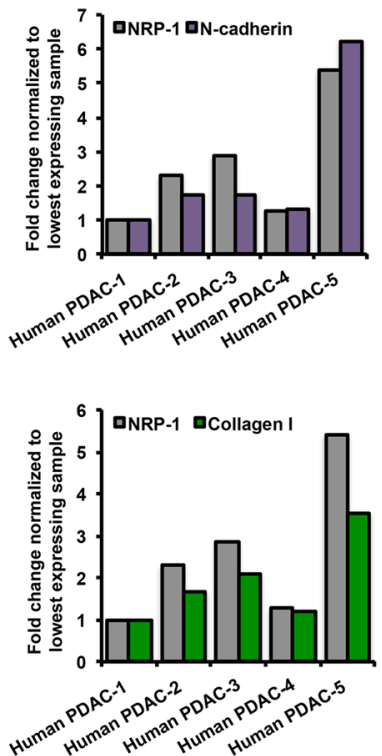

B
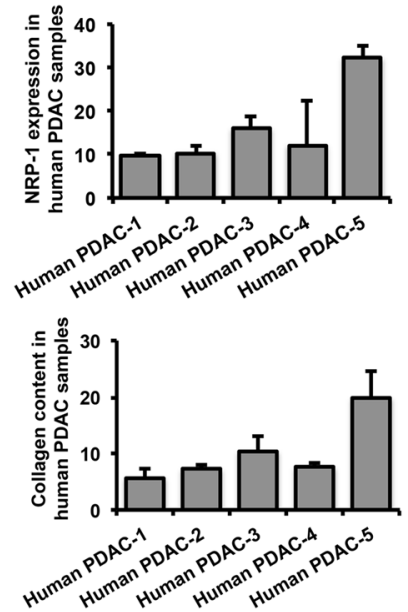

D

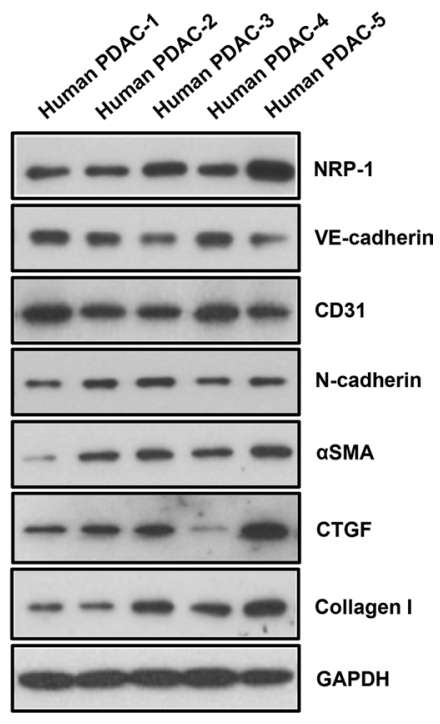

E

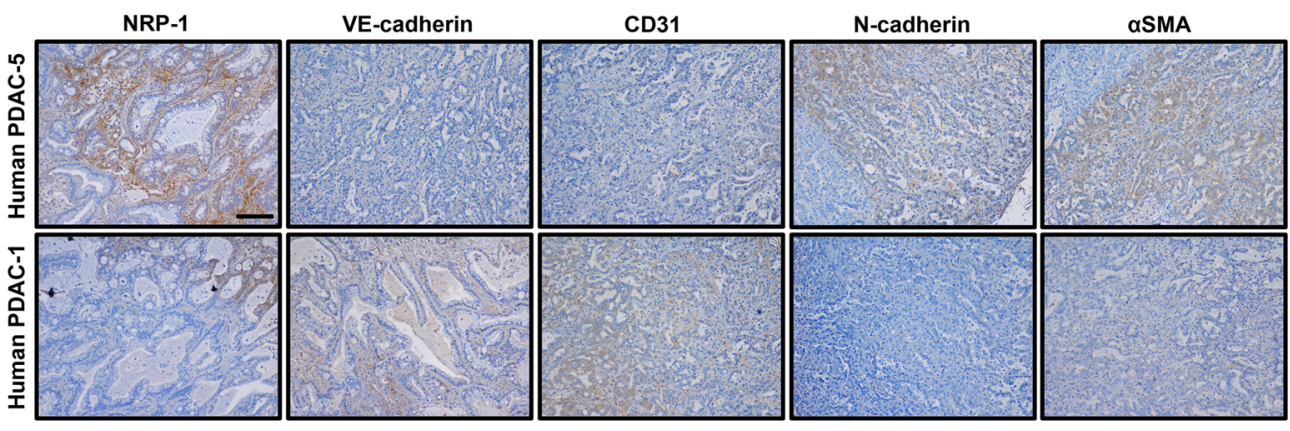

$\mathbf{F}$

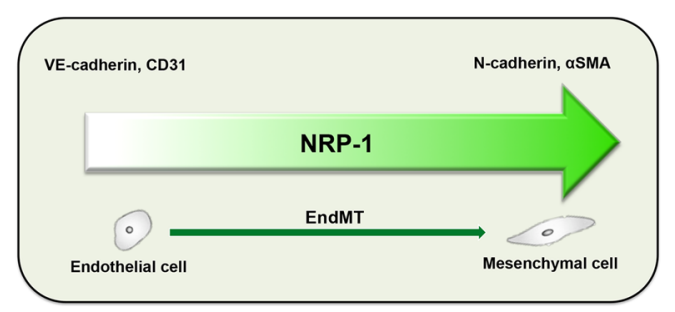

Figure 1: NRP-1 levels positively correlate with EndMT and fibrosis markers in human PDAC xenografts. Five human PDAC xenografts were obtained for this study. (A) Tumor tissues were stained with H\&E staining to recognize tissue type and 
the morphology; for NRP-1 levels (indicated by brown color) by immunohistochemistry and for extent of fibrosis by Masson's trichrome staining (indicated by blue color). Representative images reveal varying levels of tissue differentiation (Scale bar $=100 \mu \mathrm{m})$, NRP-1 expression (Scale bar $=50 \mu \mathrm{m})$ and extent of fibrosis in the xenografts (Scale bar $=100 \mu \mathrm{m})$. (B) Quantification of NRP-1 levels and collagen content using the ImageJ software (expressed in AU, arbitrary units). NRP-1 levels positively correlate with EndMT markers and pro-fibrotic genes at the transcript $(\mathbf{C})$ and protein level (D) as determined by qPCR and western blotting respectively. Correlation was determined by Spearman's Rho test (positive correlation: $0.8<\mathrm{R} \leq 1, \mathrm{R}=$ correlation coefficient). For C, data has been normalized to tissue sample having lowest expression of the respective genes that are plotted. (E) Representative immunohistochemistry images showing correlation between NRP-1 level and EndMT markers in tumor tissues having the lowest and highest NRP-1 expression as determined previously (Scale bar $=100 \mu \mathrm{m})$. (F) Summary of NRP-1-EndMT correlation.

\section{NRP-1 siRNA leads to successful knockdown at the transcript, protein and functional levels in HUVECs}

We performed siRNA-mediated knockdown of $N R P-1$ in HUVECs. In vitro knockdown studies were performed in three groups: 1 . non-transfected control, 2 . transfected scramble control and 3. transfected siNRP-1. Data from second and third groups are shown in the manuscript since data from the non-transfected control and scramble control transfected HUVECs were similar (data not shown). NRP-1 knockdown was confirmed by a significant reduction in NRP-1 expression by qPCR and immunoblotting analysis at different time points (Figure 2A, 2B). NRP-1 is primarily involved in angiogenesis through its co-receptor function with VEGFR-2 by binding to VEGF-A [44]. We therefore performed the capillary-like tube formation assay in HUVECs to assess NRP-1 knockdown at functional level and observed that siNRP-1 demonstrated a significant reduction of number of nodes and tubes as compared to scramble control (Figure 2C, 2D).

\section{Loss of NRP-1 inhibits TGFß1-induced phenotypic switching typical of endothelial-to- mesenchymal transition in HUVECs}

Light microscopy studies showed that TGF $\beta 1$ stimulated scramble-transfected HUVECs demonstrated a distinctive morphological change from typical 'cobblestone-like endothelial cell morphology' to an enlarged spindle shaped manifestation, characteristic of 'mesenchymal-like cell morphology' (Figure 2E). Additionally, we observed that these morphological changes were associated with cytoskeletal protein reorganization. In accordance, we observed an increase in $\alpha$-actinin expression and a representative mesenchymal cell-like cytoskeletal protein re-organization (Figure 2F). Interestingly, these distinctive morphological and ultrastructural protein re-organization changes were inhibited upon loss of NRP-1 (Figure 2E, 2F). Phenotypic EndMT characteristics are accompanied by corresponding changes in the expression of cell surface markers $[24,26]$. In keeping with characteristics of reversal of EndMT, upon TGF $\beta 1$ stimulation, loss of NRP-1 significantly induced the expression of endothelial markers; VE-cadherin and CD31, and inhibited the expression of mesenchymal markers; $\alpha$ SMA, $\mathrm{N}$-cadherin and transcription factor Slug as assessed by qPCR (Figure 2G), immunoblotting (Figure 2H) and immunofluorescence studies (Figure 2I) when compared to scramble control transfected cells.

Mechanistically, TGF $\beta$ signaling is crucial in regulating EndMT [36-38]. Knockdown of NRP-1 in TGF $\beta 1$-stimulated HUVECs resulted in significantly reduced expression of TGF $\beta 1$ ligand at the transcript (Figure 2J) and protein (Figure 2K) level when compared to the scramble control. The TGF $\beta 1$ isoform uses the same set of receptors as other isoforms consisting of TGFBR1 and TGFBR2 [47]. TGF $\beta 1$ binds to TGFBR2 initially, which further bind to TGFBR1 to form a complex with serine/threonine kinase activity, where TGFBR2 phosphorylates TGFBR1. This complex is further capable of phosphorylating downstream molecules belonging to the SMAD family of proteins that possess the transcription regulatory activity upon translocation to the nucleus. Knockdown of NRP-1 down-regulated TGFBR1 and TGFBR2 at transcript and protein levels as assessed by qPCR and immunoblotting, respectively, in TGF $\beta 1$ stimulated HUVECs (Figure 2J, 2K). Loss of NRP-1 was further accompanied by notably reduced SMAD2 phosphorylation as demonstrated by immunoblotting (Figure 2K, Supplementary Figure 1A, 1B). Upon investigating the expression of TGF $\beta 1$-dependent profibrotic genes, like CTGF and Collagen 1, we observed that loss of NRP-1 in TGF $\beta 1$-stimulated HUVECs significantly decreased their expression at the protein level as detected by immunoblotting (Figure 2H). As anticipated, reduction in SMAD2 phosphorylation and expression of pro-fibrotic genes was not observed in the scramble control HUVECs group (Figure 2H, 2K). Intriguingly, in the absence of TGF $\beta 1$ stimulation, loss of NRP-1 significantly increased the expression of endothelial markers; VE-cadherin and CD31, however, the decrease in expression of mesenchymal markers; $\mathrm{N}$-cadherin and $\alpha \mathrm{SMA}$, although moderately reduced, was not significant as assessed by qPCR (Supplementary Figure 1F). 
Table 1: Partial characterization of pancreatic cancer xenografts established from patients

\begin{tabular}{|c|c|c|c|c|c|c|c|}
\hline $\begin{array}{c}\text { Human PDAC } \\
\text { Xenograft }\end{array}$ & Sex & $\begin{array}{l}\text { Tumor } \\
\text { Grade }\end{array}$ & $\begin{array}{l}\text { Clinical } \\
\text { Stage }^{1}\end{array}$ & $\begin{array}{l}\text { Pathology } \\
\text { Stage }^{1}\end{array}$ & $\begin{array}{l}\text { Undergone } \\
\text { Surgery }\end{array}$ & Relapse & $\begin{array}{l}\text { Survival } \\
\text { (In days) }\end{array}$ \\
\hline 1 (OCIP23) & $\mathrm{M}$ & G3 & T3NxM0 & T3N1b & Yes & Distant & 249 \\
\hline 2 (OCIP28) & $\mathrm{F}$ & G1 & T4N0M0 & T3N0 & Yes & & $2047 *$ \\
\hline 3 (OCIP217) & $\mathrm{F}$ & G3 & T4N0M1 & T2N0 & Yes & Distant & 777 \\
\hline 4 (OCIP167) & $\mathrm{M}$ & $\mathrm{G} 2$ & $\mathrm{~T} 2 \mathrm{NxM} 0$ & T3N1b & Yes & Distant & 1150 \\
\hline 5 (OCIP232) & $\mathrm{M}$ & $\mathrm{G} 2$ & TxNxM1 & T3N1b & Yes & Distant & 681 \\
\hline
\end{tabular}

Abbreviations: M-male; F-female. Data has been adapted from Lohse et al. [85].

${ }^{1}$ TNM classification of tumors of the exocrine pancreas: T2, tumor limited to the pancreas, more than $2 \mathrm{~cm}$ in greatest dimension; T3, tumor extends directly into any of the following: duodenum, bile duct, peripancreatic tissues; T4, tumor extends directly into any of the following: stomach, spleen, colon, adjacent large vessels; N0, no regional lymph node metastasis; N1a, metastasis in a single regional lymph node; N1b, metastasis in multiple regional lymph nodes.

*Patient alive when the study was published.

Table 2: Summary of response to treatment

\begin{tabular}{|c|c|c|c|}
\hline $\begin{array}{c}\text { Human PDAC } \\
\text { Xenograft }\end{array}$ & Radio-therapy & Chemotherapy & Response to Treatment \\
\hline 1 (OCIP23) & No & Gemcitabine $^{*}$ & Progression of disease \\
\hline \multirow[t]{2}{*}{2 (OCIP28) } & Yes & Gemcitabine-Cisplatin & Partial therapeutic response \\
\hline & & Capecitabine $^{*}$ & No indication of disease \\
\hline \multirow[t]{3}{*}{3 (OCIP217) } & Yes & Gemcitabine-Cisplatin ${ }^{\#}$ & Partial therapeutic response \\
\hline & & Gemcitabine-Cisplatin $^{+}$ & Stable disease \\
\hline & & Carboplatin-paclitaxel & Progression of disease \\
\hline \multirow[t]{2}{*}{4 (OCIP167) } & No & Gemcitabine $^{*}$ & No indication of disease \\
\hline & & 5FU-irinotecan oxaliplatin ${ }^{+}$ & Stable disease \\
\hline \multirow[t]{3}{*}{5 (OCIP232) } & No & Gemcitabine* & No indication of disease \\
\hline & & $\begin{array}{l}\text { 5FU-irinotecan- } \\
\text { oxaliplatin-leucovorin }^{+}\end{array}$ & $\begin{array}{l}\text { Initial therapeutic response but } \\
\text { progressed after } 6 \text { months }\end{array}$ \\
\hline & & Veliparib $^{+}$ & Progression of disease \\
\hline
\end{tabular}

Abbreviations: 5FU- 5-fluorouracil. Data has been adapted from Lohse et al. [85].

*Adjuvant, ${ }^{\text {NNeoadjuvant, }}{ }^{+}$Palliative.

\section{Lentivirus-mediated NRP-1 transduction in HUVECs leads to successful overexpression at the transcript, protein and functional levels}

We performed lentiviral-mediated overexpression of NRP-1 in HUVECs. In vitro overexpression studies were performed in three groups; 1 . non-transduced control, 2. transduced lentiControl and 3. transduced lentiNRP-1. Data from empty control (LentiControl) and lentiNRP-1 are shown in the manuscript since data from the non-transduced control and empty control transduced HUVECs were indistinguishable (data not shown). NRP-1 overexpression was confirmed by a striking increase in NRP-1 expression by qPCR (Figure 3A) and immunoblotting (Figure 3B) analysis at different time points. The capillary-like tube formation assay exhibited that lentiNRP-1 demonstrated a significant increment in number of nodes and tubes as compared to lentiControl owing to the co-receptor function of NRP-1 during VEGF-induced angiogenesis (Figure 3C, 3D).

\section{Overexpression of NRP-1 in TGF $\beta 1$-stimlated HUVECs induces distinct morphological and molecular changes consistent with EndMT}

Light microscopy studies showed that overexpression of NRP-1 in TGF 1 -stimlated HUVECs lead to a distinctive morphological change, in accordance with phenotypic switching associated with EndMT (Figure 3E). Furthermore, we observed an increase in $\alpha$-actinin expression and a mesenchymal cell-like cytoskeletal protein 
A

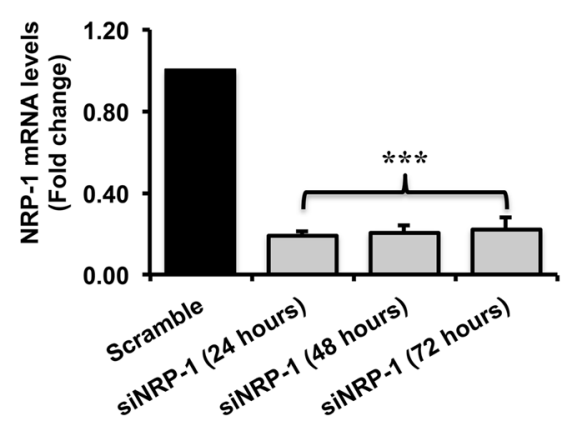

E

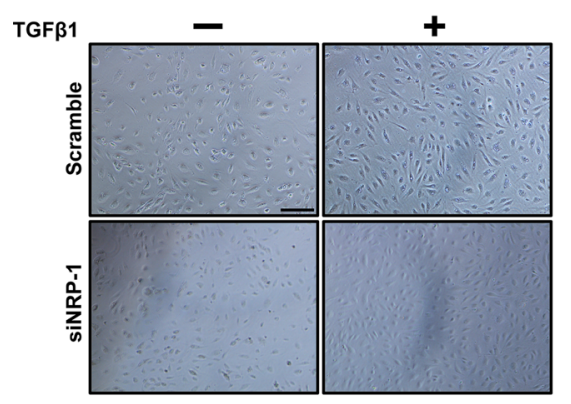

I
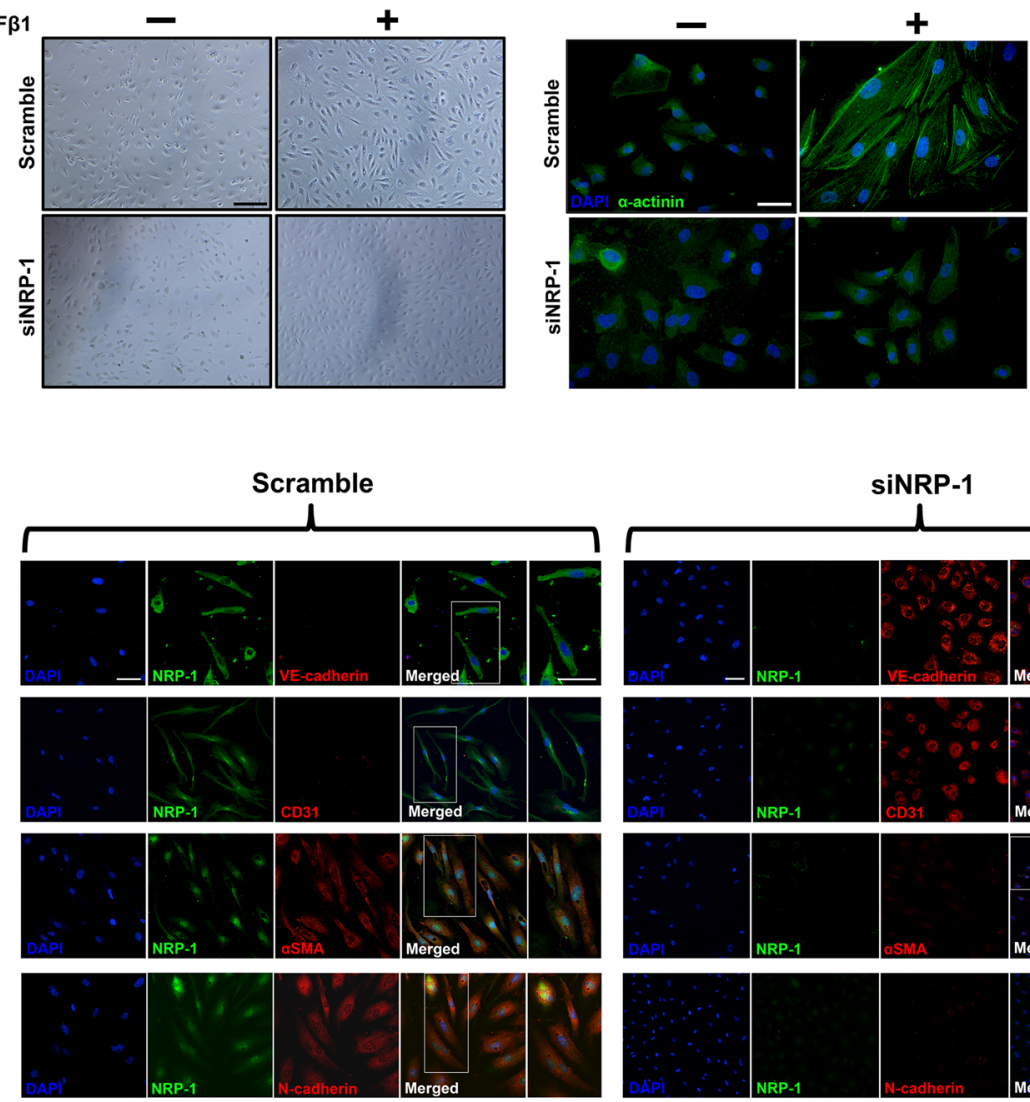

C

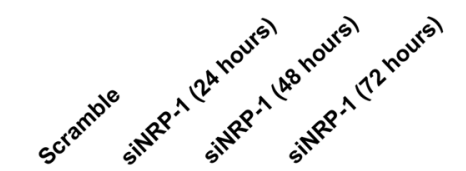

D
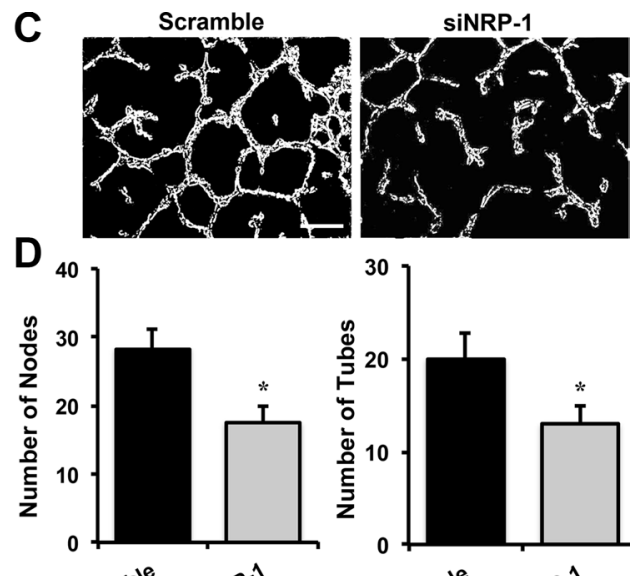

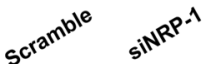

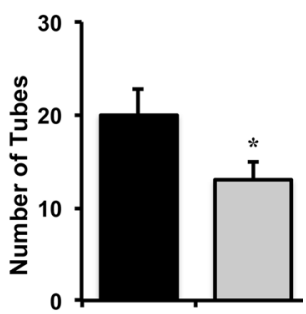

G

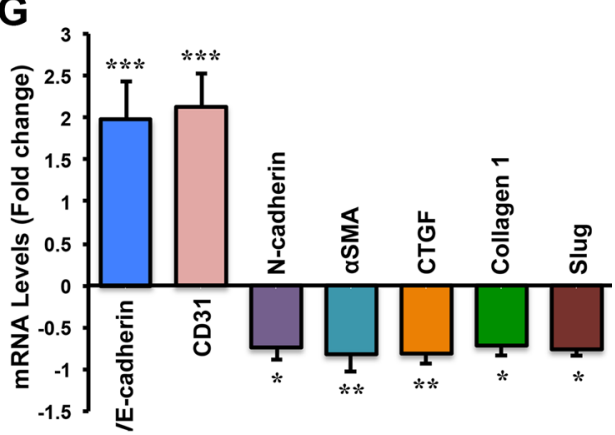

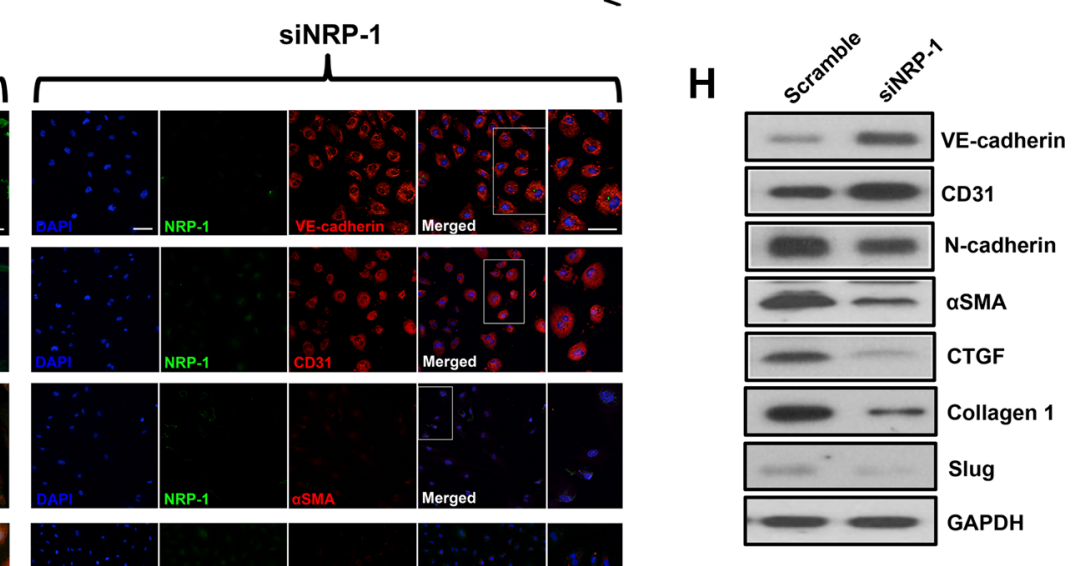

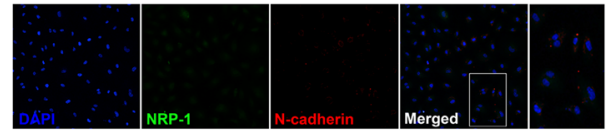

K
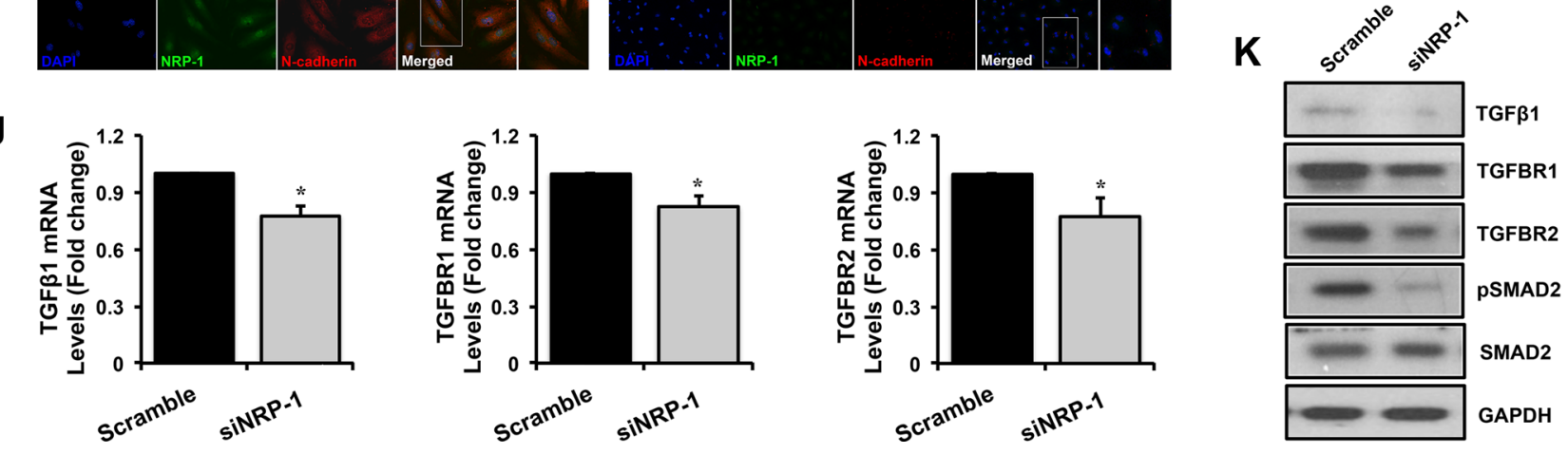

Figure 2: Loss of NRP-1 inhibits TGFß1- induced EndMT in HUVECs. HUVECs were cultured and transfected with 5 nM of either siNRP-1 or scramble control. Total RNA and protein was extracted from the transfected HUVECs at 24,48 and $72 \mathrm{~h}$ posttransfection. (A) qPCR data demonstrate successful silencing of NRP-1 ( $\sim 85 \%$ reduction) in siNRP-1 transfected HUVECs after $24 \mathrm{~h}$. All qPCR data are presented as fold change to the scrambled control. (B) NRP-1 silencing was confirmed at protein level by immunoblotting at 24, 48 and $72 \mathrm{~h}$ after transfection. GAPDH was used as a loading control. (C) Representative images from capillary-like tube formation assay demonstrating reduced number of tubes in siNRP-1 compared to scramble control at $6 \mathrm{~h}$. (D) Tube formation ability of HUVECs on 
Matrige $^{\mathrm{TM}}$ was quantified as number of nodes and number of tubes, showing that siNRP-1 caused a significant anti-angiogenic effect as compared to scramble control. ${ }^{*} p<0.05$ and ${ }^{* * *} p<0.001$ vs. scramble control. $n=5$ in triplicate. (E) Confluent monolayer of HUVECs exhibits typical cobblestone morphology in scramble control under light microscope $(20 \mathrm{X}, \mathrm{scale}=50 \mu \mathrm{m})$. TGF $\beta 1 \mathrm{stimulation}(10 \mathrm{ng} / \mathrm{ml})$ caused marked morphological changes with the cells becoming enlarged and spindle shaped in scramble control transfected cells. These morphological changes were inhibited to some extent in siNRP-1 transfected TGF $\beta 1$-stimulated HUVECs. (F) Fluorescent microscopy images $($ scale $=10 \mu \mathrm{m})$ stained with alpha-actinin ( $\alpha$-actinin; green color), demonstrating cytoskeletal protein re-organization in scramble control transfected TGF $\beta 1$-stimulated HUVECs. Nuclei were stained with DAPI (blue color). HUVECs were transfected with scramble control and siNRP-1, and total RNA and protein was extracted at $24 \mathrm{~h}$ and $48 \mathrm{~h}$, respectively. qPCR analysis demonstrates significant changes in the EndMT markers at transcript level $(\mathbf{G})$ and protein level $(\mathbf{H})$ by immunoblotting. These data were further confirmed by immunofluorescence for NRP-1 and EndMT markers (I) (scale $=20 \mu \mathrm{m}$; scale for magnified image $=10 \mu \mathrm{m}) 48 \mathrm{~h}$ post-transfection. Nuclei were stained by DAPI (blue). (J) qPCR data analysis demonstrates significant down-regulation of TGF $\beta 1$, TGFBR1 and TGFBR2 and TGF $\beta 1$-responsive genes; Slug, Collagen I and CTGF at transcript level and (K) TGF $\beta 1$, TGFBR1, TGFBR2, pSMAD2, SMAD2 and $(\mathrm{H})$ pro-fibrotic genes CTGF, Collagen I and Slug at protein level by immunoblotting upon NRP-1 silencing. ${ }^{*} p<0.05$, $* * p<0.01$, $* * * p<0.0001$ vs. scramble control. $n=3-4$ in triplicate.

re-organization upon NRP-1 overexpression as compared to lentiControl (Figure 3F). In agreement with TGF $\beta 1$ induced EndMT characteristics, NRP-1 overexpression significantly down-regulated the expression of endothelial markers; VE-cadherin and CD31, and induced the expression of the mesenchymal markers; N-cadherin, aSMA and Slug as assessed by qPCR (Figure 3G), immunoblotting (Figure $3 \mathrm{H}$ ) and immunofluorescence (Figure 3J). Interestingly, NRP-1 overexpression in the absence of TGF $\beta 1$ stimulation resulted in an increase in mesenchymal markers' expression but no significant decrease in the expression of all the assessed endothelial cell markers (Supplementary Figure 1G).

Examining at the mechanistic level, we observed that overexpression of NRP-1 in TGF $\beta 1$-stimulated HUVECs resulted in significantly elevated expression of TGF $\beta 1$ at the transcript and protein level when compared to lentiControl (Figure 3J, 3K). Additionally, overexpression of NRP-1 up-regulated TGFBR1 and TGFBR2 at transcript and protein level as assessed by qPCR (Figure 3J) and immunoblotting (Figure $3 \mathrm{~K}$ ) respectively, and led to increased SMAD2 phosphorylation as demonstrated by immunoblotting (Figure 3K). Overexpression of NRP-1 along with TGF $\beta 1$-stimulation significantly augmented the expression of TGF $\beta 1$-dependent pro-fibrotic genes, like CTGF and Collagen 1 at transcript and protein level as compared to lentiControl (Figure 3G, 3H). Thus our NRP-1 overexpression study was antagonistic with our loss of NRP-1 function study, and in accordance with our hypothesis. This confirms the novel in vitro regulatory role of NRP-1 in TGF $\beta 1$-induced EndMT.

\section{Loss of NRP-1 inhibits EndMT and fibrosis in vivo and results in reduced tumor growth}

Prior to in vivo studies, the knockdown efficiency of the shNRP-1 minicircle was tested in vitro in BxPC-3 cells. NRP-1 knockdown was confirmed by a significant reduction in NRP-1 expression by qPCR and immunoblotting analysis at different time points (Supplementary Figure 1C, 1D). Moreover, NRP-1 knockdown significantly affected the growth kinetics of BxPC-3 cancer cells in vitro as observed by the MTT assay for cell viability (Supplementary Figure 1E). Orthotopic pancreatic tumors were grown in athymic rats and followed till day 56 after implantation surgery. Ultrasound-targeted microbubble destruction (UTMD) of shNRP-1 minicircle was performed for NRP-1 silencing at day 28 post-implantation in a subset of animals. Total RNA and protein were extracted from the tumor tissues. qPCR data (Figure 4A) and immunoblotting data (Figure 4B) demonstrate successful silencing of NRP-1 in shNRP-1 minicircle delivered nude rats. Tumor tissues obtained from gene delivered animals were stained with $H \& E$ to characterize tissue type and morphology (Figure 4C) and extent of fibrosis by Masson's trichrome staining. We observed a typical ductal morphology and increased collagen content, features consistent with the characteristics of PDAC. Histological assessments further revealed reduced NRP-1 expression and reduced extent of fibrosis in shNRP-1 minicircle delivered animals (Figure 4C). Investigating the role of EndMT in tumor fibrosis, our qPCR analysis demonstrated significant changes in the EndMT markers at transcript level (Figure 4A) and protein level (Figure 4B) in shNRP-1 minicircle delivered nude rats when compared to scramble minicircle delivered rats. These data were further confirmed by immunohistochemistry for NRP-1 and EndMT markers (Figure 4C). At day 56, we observed significant reductions in tumor volumes in shNRP-1 delivered animals compared to scramble treated group, as measured by volumetric assessment of explanted tumors (Figure 4D). Representative CEU perfusion images of orthotopic pancreatic tumors on day 28 (before gene delivery) and day 56 (28 days after gene delivery) are shown in Figure 4E. CEU assessments at day 28 providing a pre-delivery baseline assessment, demonstrated no significant differences in normalized microvascular blood volume (MBV) and microvascular blood flow (MBF) between different tumors (Figure 4G, 4H). For perfusion imaging, the blood pool signal measured from a region of interest placed in the left ventricular (LV) cavity of 
A

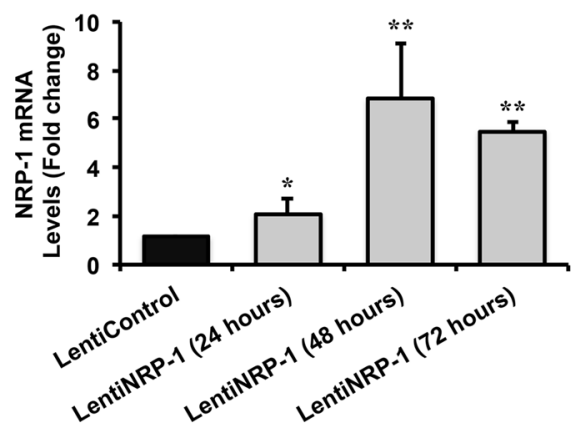

E

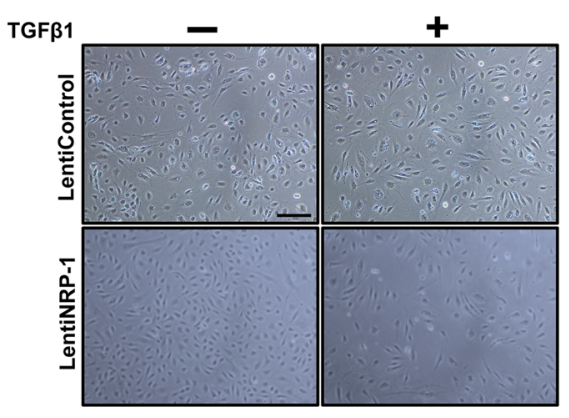

I
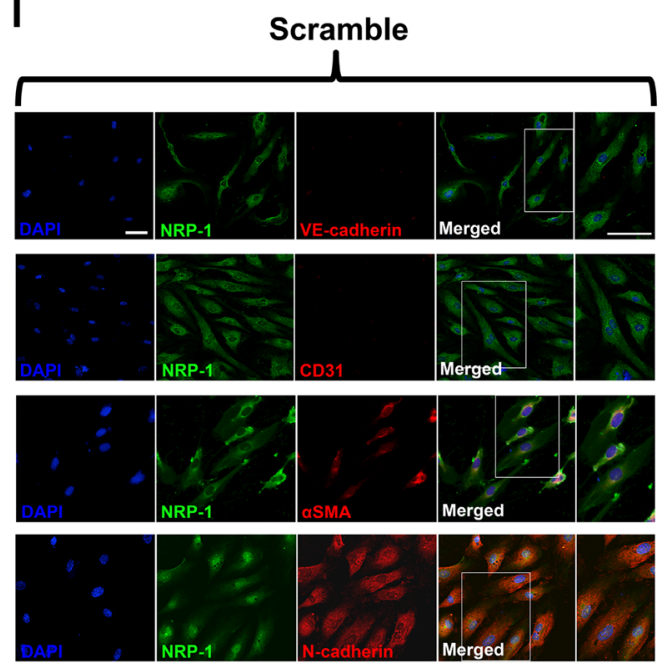

J

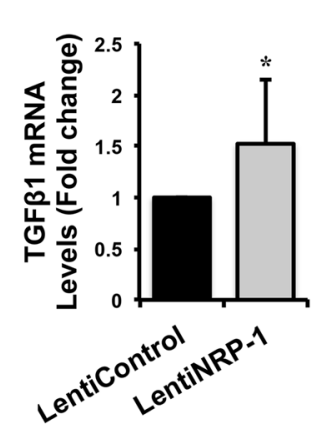

B

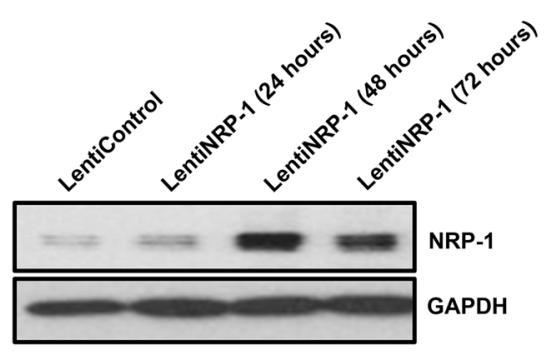

F
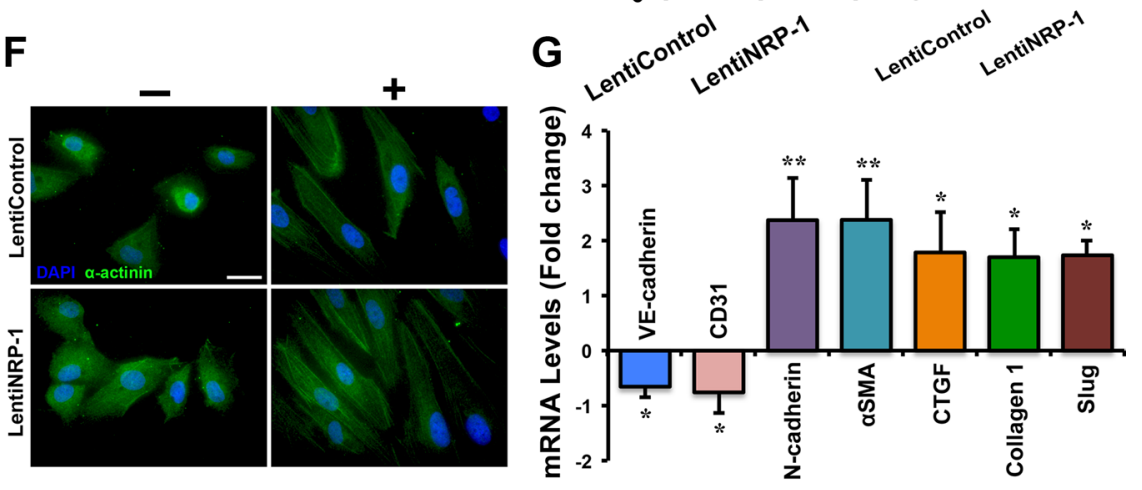

H
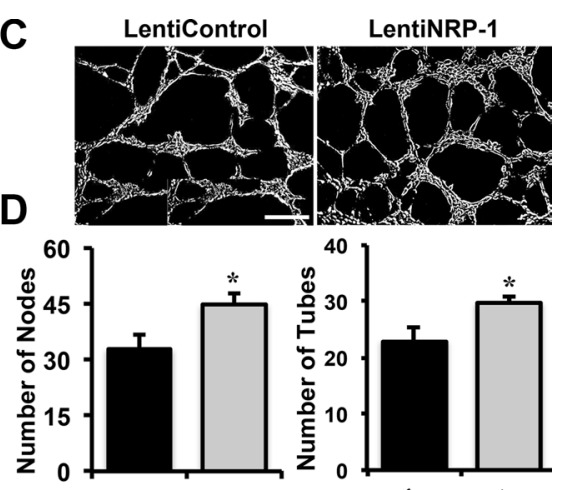
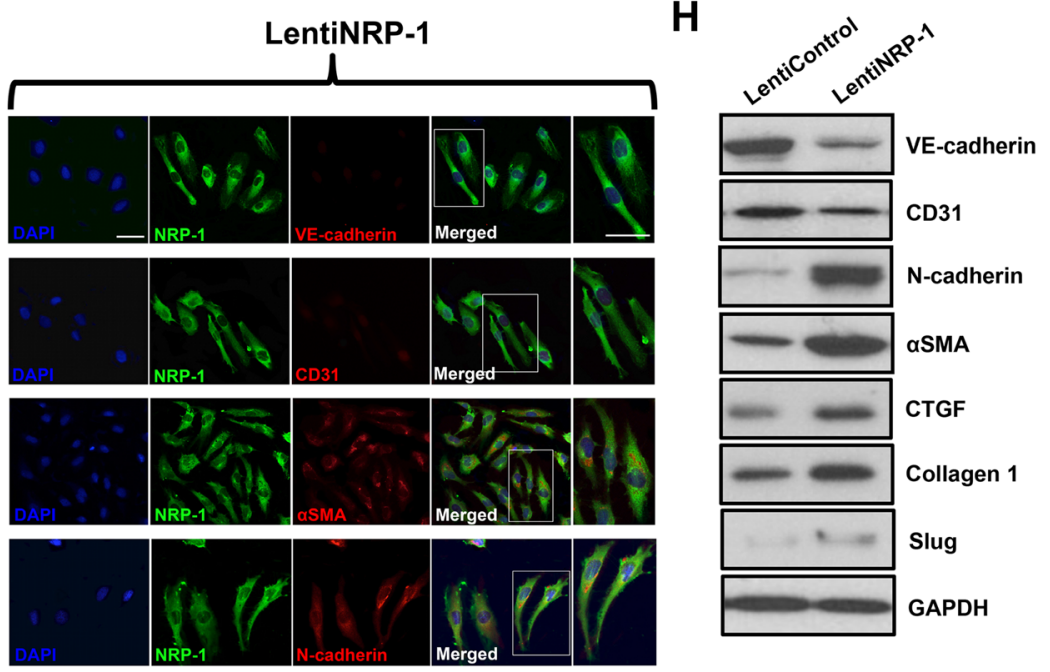

K

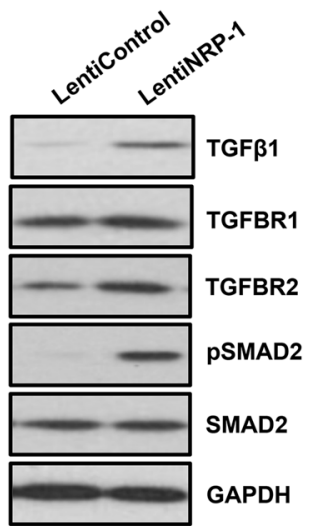

Figure 3: Lentivirus-mediated NRP-1 overexpression exacerbates TGFß1-induced EndMT in HUVECs. HUVECs were cultured and transduced with either lentiNRP-1 or blank/empty control lentivirus (lentiControl). Total RNA and protein was extracted from the transduced HUVECs at 24, 48 and $72 \mathrm{~h}$ post-transduction. (A) qPCR data demonstrate successful overexpression of NRP-1 in lentiNRP-1 transduced HUVECs after $48 \mathrm{~h}$. All qPCR data are presented as fold change to the scrambled control. (B) NRP-1 overexpression 
was confirmed at protein level by immunoblotting. (C) Representative images from capillary-like tube formation assay demonstrating increased number of tubes in lentiNRP-1 compared to lentiControl at $6 \mathrm{~h}$ time point. (D) Tube formation ability of HUVECs on Matrigel ${ }^{\mathrm{TM}}$ was quantified as number of nodes and number of tubes and showed that lentiNRP-1 caused a significant pro-angiogenic effect as compared to lentiControl. ${ }^{*} p<0.05$ and $* * p<0.01$ vs. lentiControl. $n=5$. (E) Confluent monolayer of HUVECs exhibits typical cobblestone morphology in scramble control under light microscope (scale $=50 \mu \mathrm{m})$. TGF $\beta 1$ stimulation caused marked morphological changes with the cells becoming enlarged and spindle shaped in cells transduced with lentiControl. Similar morphological changes were observed in lentiNRP-1 transduced HUVECs upon TGF $\beta 1$ stimulation. (F) Fluorescent microscopic images (scale $=10 \mu \mathrm{m}$ ) stained with alpha-actinin ( $\alpha$-actinin; green color), demonstrating cytoskeletal protein re-organization in lentiNRP-1 transduced HUVECs. Nuclei were stained with DAPI (blue color). HUVECs were transduced with lentiControl and lentiNRP-1, and total RNA and protein was extracted at 48 h. (G) qPCR analysis demonstrates significant changes in the EndMT markers at transcript level and protein level by immunoblotting (H). (I) These data were further confirmed by immunofluorescence for NRP-1 and EndMT markers (Scramble: scale $=20 \mu \mathrm{m}$, scale for magnified image $=10 \mu \mathrm{m}$; LentiNRP-1: scale $=10 \mu \mathrm{m}$, scale for magnified image $=10 \mu \mathrm{m}) 48 \mathrm{~h}$ post-transduction in the photomicrographs. Nuclei were stained by DAPI (blue). (J) qPCR data analysis demonstrates significant down-regulation of TGF $\beta 1$, TGFBR1, TGFBR2 and (G) TGF $\beta 1$-responsive genes Slug, Collagen 1 and CTGF at transcript level and (K) TGF $\beta 1$, TGFBR1, TGFBR2, pSMAD2, SMAD2 and (H) pro-fibrotic genes Slug, Collagen I and CTGF at protein level by immunoblotting upon NRP-1 overexpression. Nuclei were stained by DAPI (blue) $* p<0.05, * * p<0.01$ and $* * * p<0.001$ vs. lentiControl. $n=3-4$ in triplicate.

the heart was used to normalize tumor plateau acoustic signal for systemic microbubble concentration [69]. Tumor area (Figure 4F) and perfusion (Figure 4G, 4H) was found to be greater and more diffuse throughout the tumor in scramble minicircle delivered rats, while reduced perfusion was observed in tumors treated with shNRP-1 minicircle. At day 56, the plateau signal intensity from tumors, representing $\mathrm{MBV}$, was markedly reduced in the group treated with shNRP-1 minicircle as compared with scramble minicircle (Figure 4G). Moreover, tumor MBF was moderately reduced in the group treated with shNRP-1 minicircle as compared with scramble minicircle (Figure 4H). Given the transfection efficacy of ultrasoundmediated gene delivery coupled with delivery of high in vivo transfection efficiency of minicircle vectors, we would expect the shRNA knockdown to last up to 4-6 weeks in our animals. Day 56 in our study is 28 days after gene delivery, thus our significant knockdown of NRP-1 at the mRNA and protein level as consistent with prior studies using minicircle DNA vectors [70, 71]. We cannot exclude the possibility that NRP-1 expression would have been re-gained by certain cell populations by this time, as transfection is not equal in each and every cell type. However, we observed significant knockdown of NRP-1 till day 28 presumably due to high transfection efficiency of shRNA-minicircle and ultrasound mediated delivery.

\section{DISCUSSION}

Pancreatic ductal adenocarcinoma remains the most malignant and common type of pancreatic cancer. Although considered rare in terms of incidence, it remains the 5th cause of cancer related deaths, with the lowest five-year survival rate $[1,5]$. Some of the important characteristics include; lack of early clinical symptoms and poor prognosis, multifaceted and complex genetic alterations and high incidences of distant metastases that contribute to the low median survival. Morphologically, PDAC is characterized by a dense fibrotic reaction termed as desmoplasia $[14,17$, 18]. While previously considered to be benign, this unique compartment within the tumor has been shown to contribute to several events that initiate and promote carcinogenesis. In fact about $80-90 \%$ of PDAC tumor volume consists of this cancer-supportive compartment. Treating these patients becomes challenging owing to this dense stromal barrier, primarily composed of fibroblasts, making anti-fibrotic therapy a potential treatment modality.

The origin of CAFs, contributing to the formation of dense stroma, is a subject gaining importance lately. These fibroblasts are responsible for releasing potentially oncogenic signals, such as TGF $\beta$, VEGF and other growth factors. Among several potential mechanisms for their origin, EndMT has gathered attention recently $[34,35]$. Although first described during embryonic heart development, recent data suggest that maladaptive EndMT plays a major role in several fibrotic pathological disorders including pulmonary fibrosis, renal fibrosis and cancer [29-33, 39]. Loss of endothelial cell surface markers and gain of mesenchymal cell markers represent the hallmark of this phenomenon. Notably, EndMT is a multifactorial process driven by molecular mediators like TGF $\beta 1$. Therefore, investigating the role of novel biological clues and targets regulating EndMT can provide mechanistic insights into the underlying molecular processes involved in PDAC progression. More importantly, validation of such targets can lead to potential treatment options for fibrosisrelated disorders, including PDAC. Here, we highlight EndMT as a potential source of CAFs in PDAC, with a discussion of proposed role of an angiogenic co-receptor, putative mechanisms and therapeutic implications.

The role of neuropilins in carcinogenesis, particularly the NRP-1 isoform, has been well documented [43]. Originally discovered for its role during embryonic nervous system development, the discovery of its function as a co-receptor for VEGFR-2 during angiogenesis [49] prompted investigators to study its function in tumor angiogenesis and carcinogenesis. Subsequently, several 
A

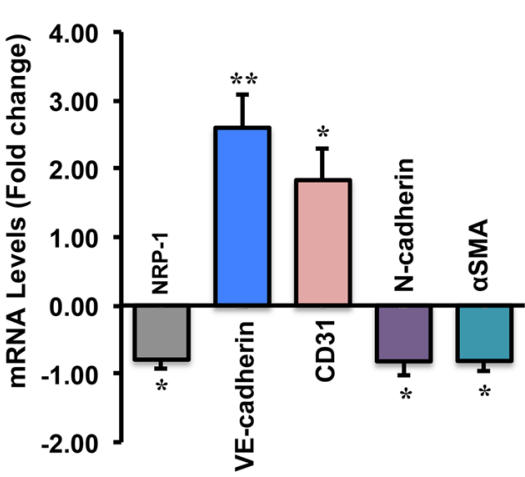

B

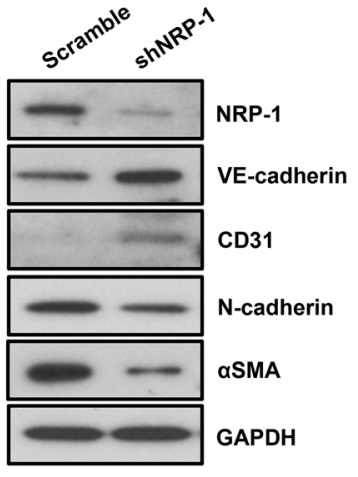

C

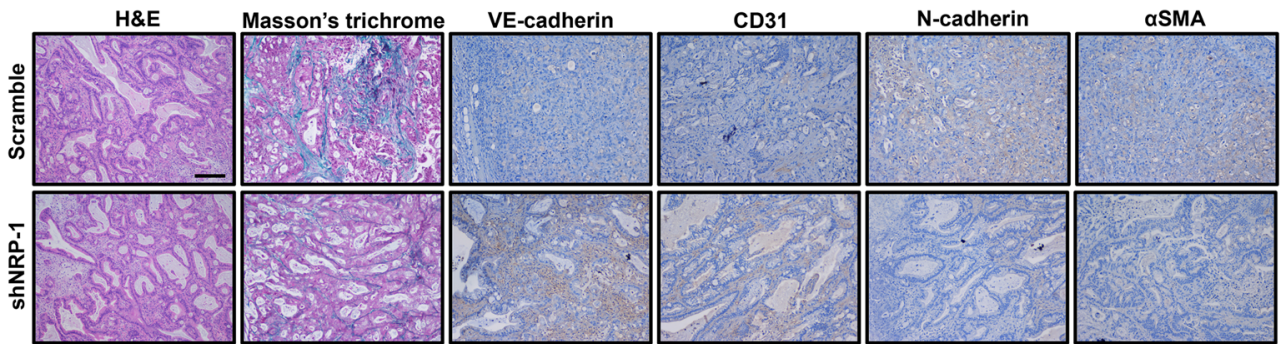

D

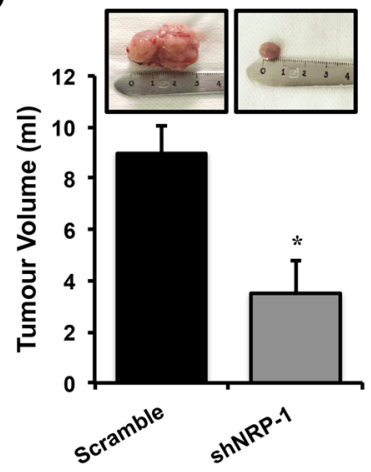

G

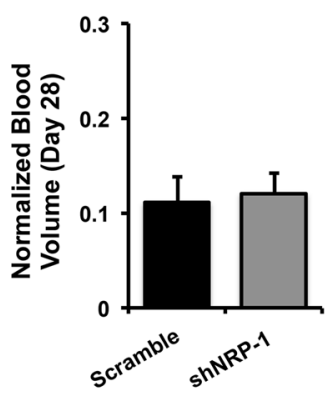

$\mathbf{E}$

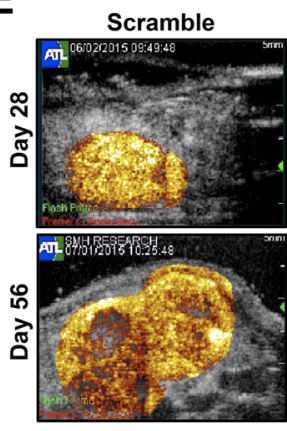

$\mathrm{H}$

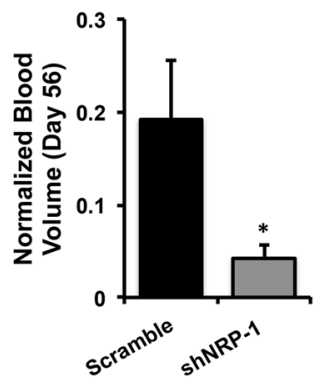

$\mathbf{F}$
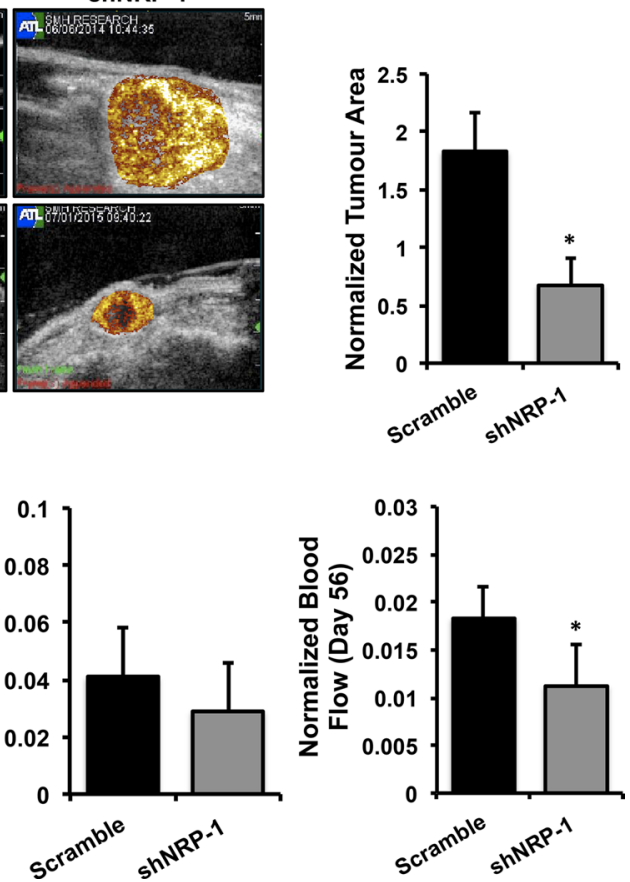

Figure 4: Loss of NRP-1 inhibits EndMT and fibrosis in vivo and results in reduced tumor growth. Total RNA and protein was extracted from the tumor tissues. (A) qPCR data and (B) immunoblotting demonstrate successful silencing of NRP-1 in shNRP-1 minicircle delivered animals. (A) qPCR analysis demonstrates significant changes in the EndMT markers at transcript level and protein level (B) by immunoblotting. (C) These data were further confirmed by immunohistochemistry for NRP-1 and EndMT markers (scale $=100 \mu \mathrm{m}$; indicated by brown color). The tumor tissues were also stained with $\mathrm{H} \& \mathrm{E}$ to recognize tissue type and the morphology, and extent of fibrosis by Masson's trichrome staining (indicated by blue color). (C) Representative images reveal ductal morphology, reduced NRP-1 levels and reduced extent of fibrosis in in shNRP-1 minicircle delivered animals. (D) Tumor volumes (ml) as measured by volumetric assessment, with smallest tumor volumes seen in the shNRP-1 minicircle group as compared to scramble group. (E) Representative contrast-enhanced ultrasound perfusion images of orthotopic pancreatic tumors on day 28 (before gene delivery) and day 56 (28 days after gene delivery). Images are color coded, with bright yellow/orange signifying the highest acoustic signal and greatest perfusion. Tumor area $(\mathbf{F})$ and perfusion $(\mathbf{G}, \mathbf{H})$ is greater and more complete throughout the tumor in scramble minicircle group, while reduced perfusion seen in tumors treated with shNRP-1 minicircle. (G,H) At day 28 there were no significant differences in microvascular blood volume (MBV) and microvascular blood flow (MBF) between groups. Normalized tumor blood volume $(\mathrm{G})$ and blood flow $(\mathrm{H})$ at day 56. At day 56, tumor blood volume and flow was significantly reduced in shNRP-1 minicircle group compared with scramble control group. ${ }^{*} p<0.05,{ }^{* *} p<0.01$ vs. scramble. $n=4$ for each group. Tumor area and volume data are expressed as mean $\pm \mathrm{SEM}$. 
studies highlighted that NRP-1 was aberrantly expressed in several cancer types, including PDAC, and was involved in a plethora of cancer initiating and promoting pathways due to its distinctive ability to bind numerous growth factors and ligands $[44,45,50-53,60,61]$. Moreover, the ability of NRP-1 to exert co-receptor function to TGF $\beta 1$ opened new realms for investigation into oncogenic processes like EMT [51, 56-58]. Together, these NRP- 1 findings in conjunction with studies highlighting the role of TGF $\beta$ signaling in EndMT, helped us postulate that NRP-1 could be playing a previously unrecognized regulatory role in TGF $\beta 1$-induced EndMT and potentially contributes towards tumor fibrosis.

Our correlation data obtained from human PDAC xenografts, in addition to in vitro knockdown and overexpression studies, led to an important observation in this study; NRP-1 plays a previously undetermined regulatory role in TGF $\beta$-induced EndMT and associated fibrosis. Indeed, knockdown of NRP-1 inhibited, while overexpression of NRP-1 promoted EndMT and associated fibrosis. Our studies on human PDAC tissues in this report demonstrated a positive correlation between NRP-1 levels, EndMT markers and pro-fibrotic genes' expression (Figure 1A-1E). We observed that NRP-1 was expressed in all the tissue samples, however, with varying degrees. Moreover, the fibrosis and immunohistochemistry quantification results confirmed the significant correlation between NRP-1 levels and the extent of fibrosis (Figure 1A, 1B). These promising human data prompted us to specifically focus further on the role of NRP-1 in EndMTinduced tumor fibrosis. To our knowledge, this is the first study to report a novel function of NRP-1 in modulating TGF $\beta 1$-induced EndMT and associated tumor fibrosis.

By measuring the NRP-1 transcript and protein expression along with the assessment of its in vitro angiogenic role, we confirmed success of our NRP-1 knockdown (Figure 2A-2D) and overexpression (Figure 3A-3D) studies in HUVECs. Furthermore, we observed that loss of endothelial NRP-1 repressed EndMT characteristics. TGF $\beta 1$-mediated distinct morphological changes from cobblestone-like endothelial cell morphology to an enlarged spindle shaped, fibroblast like morphology (Figure 2E) and cytoskeletal protein organizational changes were inhibited upon NRP-1 silencing (Figure 2F). These microscopic modifications were in complete accordance with the changes in transcript (Figure 2G) and protein (Figure 2H, 2I) expression of endothelial (VE-cadherin and CD31) and mesenchymal (N-cadherin and $\alpha \mathrm{SMA}$ ) cell markers. Several evidences have highlighted the role of TGF $\beta 1$ signaling as a primary mediator in EndMT [36, 37, 39]. Therefore, we assessed this signaling pathway following NRP-1 silencing and overexpression. HUVEC-specific loss of NRP-1 downregulates and deactivates TGF $\beta$ signaling, culminating in reduced phosphorylated SMAD-mediated Slug, CTGF, and Collagen 1 transcription (Figure 2H, 2K). Reduction in Slug expression in turn up-regulates the expression of the endothelial markers VE-cadherin and CD31, which inhibits the EndMT process. Furthermore, decreased levels of NRP-1 led to reduced TGF $\beta$ signaling and associated expression of TGF $\beta$-responsive profibrotic genes (Figure 2H, 2J, 2K). As anticipated, the overexpression studies demonstrated contrasting results when compared to the knockdown studies. NRP-1 overexpression promoted EndMT associated phenotypic switching in addition to the corresponding changes in expression of cell surface markers (Figure 3E-3I). Additionally, increased levels of NRP-1 led to an elevated TGF $\beta$ signaling and associated expression of TGF $\beta$ responsive pro-fibrotic genes (Figure $3 \mathrm{H}, 3 \mathrm{~J}, 3 \mathrm{~K}$ ). In contrast to TGF $\beta 1$ stimulation combined with NRP-1 overexpression, NRP-1 when overexpressed in the absence of TGF $\beta 1$ was unable to facilitate the complete transitioning of endothelial cell into mesenchymal cell. The reason behind this phenomenon could be that the regulatory role of NRP-1 in EndMT is not independent of TGF $\beta 1$. Thus our knockdown and overexpression studies provide, for the first time, solid evidence of the involvement of NRP-1 in regulating TGF $\beta 1$-induced EndMT as a potential source of CAFs. Recently, in a study of sprouting angiogenesis Aspalter et al. reported, using loss- and gain-of-function experiments that NRP-1 negatively regulated SMAD2/3 activation downstream of TGF $\beta 1$ and Bmp9 signaling [72]. In another study, Hirota et al. reported that NRP-1 and $\beta 8$ integrin cooperatively balanced TGF $\beta$ signaling in brain endothelial cells. Particularly, shNRP-1 produced enhanced pSMAD3 levels upon TGF $\beta$ stimulation [73]. These studies contrast with our current findings showing that NRP-1 enhances canonical TGF $\beta$ signaling in the process of EndMT, and suggest that the function of NRP- 1 differs between EndMT and sprouting angiogenesis. The response of endothelial cells to TGF $\beta$ is extremely complex and can occur through two antagonistic pathways: ALK5/ SMAD2,3 or ALK1/SMAD1,5. In the case of TGF $\beta$ 's failure to induce canonical SMAD2/3 signaling, TGF $\beta$ may interact with ALK1 (type I receptor) and activate SMAD1 and SMAD5. In endothelial cells, this leads to an increase in cell proliferation and migration [74]. Additionally, in the absence of canonical signaling, non-SMAD (non-canonical) pathways are also activated by TGF $\beta$ through either phosphorylation or direct interaction of its receptors. These non-SMAD pathways include various branches of MAP kinase (MAPK) pathways, Rho-like GTPase signaling pathways, and phosphatidylinositol-3-kinase (PI3K)/AKT pathways [75]. Although the activation of these other pathways cannot be ruled out, our observations regarding reduced TGF $\beta$ ligand levels upon NRP-1 silencing prompted us to mainly focus on the EndMT relevant canonical SMAD2/3 pathway. Furthermore, neuropilins can also promote non-canonical TGF $\beta$ signaling [76] and the activation of these non-canonical pathways can suppress canonical signaling [43]. These pathway interactions might be of 
importance in the angiogenesis studies outlined above. We hypothesize that the endothelial cell response in the NRP-1/TGF $\beta$ interaction is highly context dependent, and these cells respond differently under different physiological/pathological conditions.

To directly link the preliminary in vitro knockdown studies with the pathobiology of tumor fibrosis, we further tested the effects of NRP-1 silencing in a clinically relevant orthotopic model of PDAC in athymic rats. This cell line can also be directly injected into the pancreas to establish orthotopic tumors [77-79]. However, we opted for the surgical implantation technique to establish our orthotopic tumors, as implanted tumors closely mimic the clinical course of the disease observed in human pancreatic cancer patients. We also preferred the implantation technique because direct injection of cells into the pancreas is sometimes associated with possible leakage, failure of primary tumor establishment and inconsistent metastatic patterns. The rats were delivered with shRNA-minicircle DNA vector targeted against NRP-1 using UTMD 28 days after tumor implantation, a validated noninvasive technique for gene delivery [80-82]. In our study we observed that at day 28 post-implantation, the tumor attained a size that was easily detectable by contrast enhanced ultrasound. We recorded baseline tumor perfusion at day 28 that was subsequently used for normalization to the perfusion recorded at day 56 . This was important in our study as normalization ruled out the size/perfusion variations observed between different animals at the time of gene delivery and gave us an accurate estimate of size and perfusion reductions after gene delivery. Twenty-eight days after shNRP-1 minicircle delivery, rats demonstrated modest but significant NRP-1 knockdown along with basal increase in the endothelial markers, down-regulation of mesenchymal markers and TGF $\beta 1$-responsive Slug and CTGF expression (Figure 4A, 4B, 4C) and reduced tumor fibrosis (Figure 4C). Furthermore, the shNRP-1 delivered animals demonstrated a modest reduction in tumor volume, tumor area, microvascular blood volume and blood flow, compared to the scramble group (Figure 4D-4H). These in vivo results suggest that loss of NRP-1 could potentially lead to reduced tumor growth, angiogenesis and fibrosis possibly through limiting EndMT as well as reducing NRP-1VEGFR-2 signaling. We did not examine longer time points for longevity or survival advantage of NRP1 knockdown. Survival analysis by Kaplan-Meier estimates will be a focus of our future studies along this line of investigation. A putative mechanistic schematic representation is shown to summarize the effect of endothelial cell loss and gain of NRP-1 on EndMT and tumor fibrosis (Figure 5).

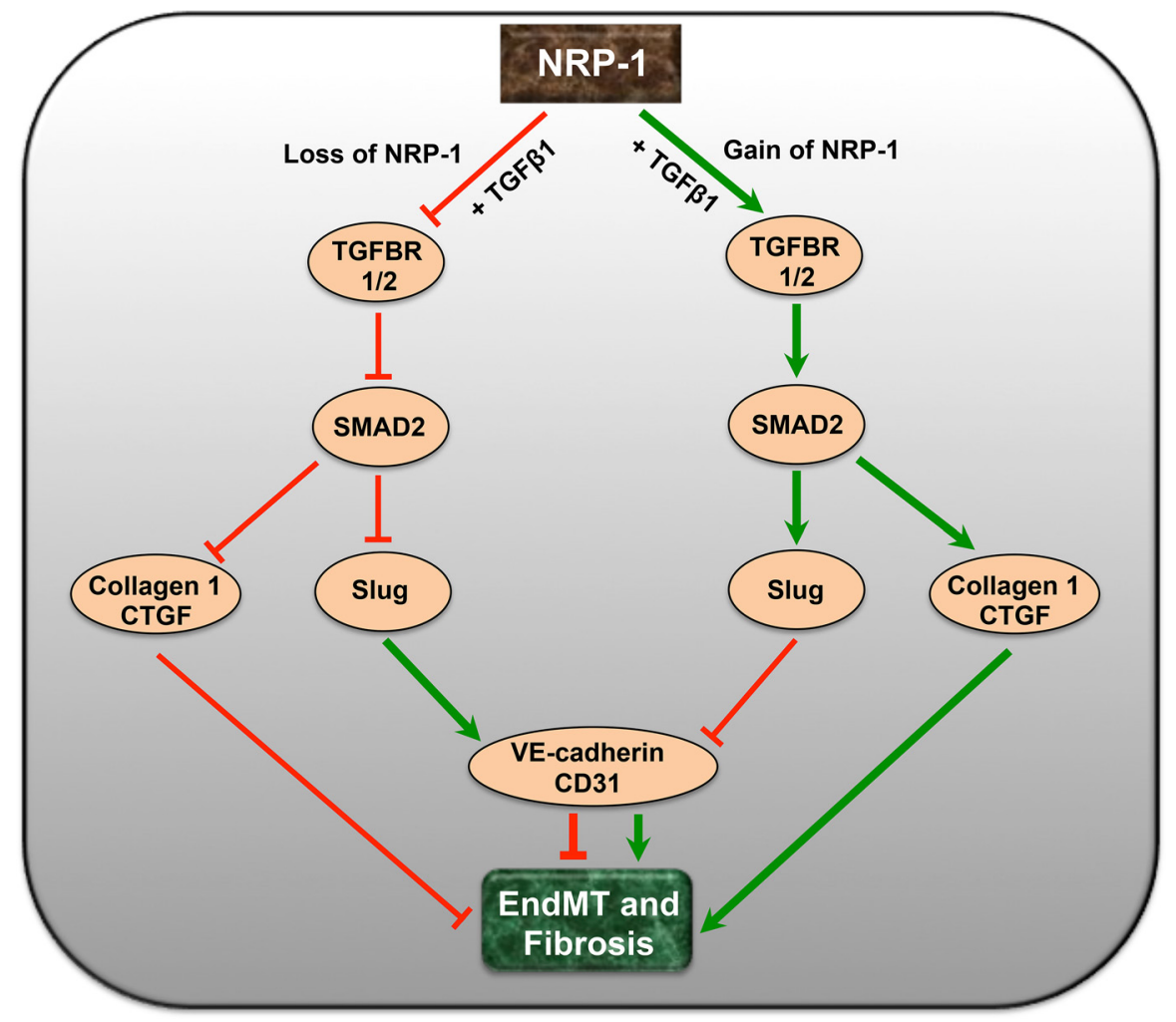

Figure 5: A putative schematic diagram depicting the complex interaction of NRP-1 and TGF $\beta$-signaling pathway in EndMT and tumor fibrosis. Endothelial cell-specific loss of NRP-1 down-regulates and deactivates TGF $\beta$ signaling and its receptors leading to reduced pSMAD2, Slug, CTGF and Collagen 1. The reduction in the levels of transcription factor Slug further up-regulates the expression of endothelial markers like VE-cadherin and CD31, limiting EndMT. Contrastingly, overexpression of NRP-1 exacerbates the TGF $\beta$-induced EndMT and associated tumor fibrosis. 
Our in vitro and in vivo studies have further validated that TGF $\beta 1$-induced EndMT can be modulated in response to manipulations of NRP-1 levels. In our experiments, we also observed a critical role of the transcription factor Slug that exacerbates the process of EndMT as previously published [39]. The exact role of other signaling pathways and transcription factors is not entirely clear at this point and warrants further mechanistic work. The recent discoveries of EndMT in various pathologies, including cancer, suggest that modulating EndMT may represent a promising novel treatment option. Studies have demonstrated that NRP-1 knockdown increases chemosensitivity towards chemotherapeutic drugs in a variety of cancer cells, including pancreatic cancer cells $[61,83]$. Of interest, recently Zheng et al. highlighted the role of EMT in drug resistance and the potential of combining EMT inhibition with chemotherapy for the treatment of pancreatic cancer [84]. However, the exact contribution of EndMT towards drug resistance and the mechanistic role of NRP-1 in EndMT-dependent chemosensitivity are unknown. Moreover, the therapeutic benefit of inhibiting NRP-1-mediated EndMT combined with chemotherapy necessitates further investigation.

In conclusion, we provide herein for the first time, solid evidence of a novel anti-NRP-1 therapy that could lead to a reduction in pancreatic tumor fibrosis and growth via a regulatory effect on TGF $\beta 1$-induced EndMT. We believe that therapies directed at targets like NRP-1 to inhibit EndMT have tremendous translational implications as they could delay PDAC tumor progression, possibly owing to impaired angiogenesis as well as limited CAF recruitment.

\section{MATERIALS AND METHODS}

\section{Cell culture and RNA interference}

Human umbilical vein endothelial cells (HUVECs, Lonza) were grown in endothelial cell growth medium-2 (EGM ${ }^{\text {TM}}-2$ Bulletkit ${ }^{\mathrm{TM}}$; Lonza) containing growth factors, serum and antibiotics. SiRNA-mediated NRP-1 knockdown studies were performed with $5 \mathrm{nM}$ siNRP-1 or scramble control (Ambion) and the Dharmafect-4 transfection reagent (Dharmacon) following the manufacturer's guidelines. Recombinant human TGF $\beta 1$ (R\&D Systems) was used for the TGF $\beta 1$-induced EndMT assays at a concentration of $10 \mathrm{ng} / \mathrm{ml}$.

\section{Lentivirus-mediated NRP-1 overexpression studies}

HUVECs were transduced with lentiNRP-1 or Empty (referred to as lentiControl) vectors (Applied Biological Materials) according to manufacturer's protocols and cells were cultured as described above.

\section{Quantitative real time - polymerase chain reaction (qPCR)}

HUVECs were cultured with either siNRP-1, lentiviral-NRP-1 or respective controls and at 24, 48 and $72 \mathrm{~h}$ post-transfection/transduction, total RNA was extracted from HUVECs using TRIzol ${ }^{\circledR}$ reagent (Invitrogen). Complementary DNA (cDNA) was synthesized using iScript cDNA Synthesis Kit (Bio-Rad) and qPCR was performed using SYBR Green (Quanta Biosciences) following manufacturer's protocols. Gene expression assays were performed with forward and reverse primers for NRP-1, CD31, VE-cadherin, $\alpha S M A$ ( $\alpha$-Smooth Muscle Actin), N-cadherin, Slug, TGFß1, TGFBR1, TGFBR2, Collagen 1, CTGF (Connective Tissue Growth Factor) and GAPDH (Supplementary Table S1). Data analysis was performed using manufacturer's integrated web-based software package (StepOne software v2.1) with cycle threshold $(\mathrm{Ct})$-based fold-change calculations.

\section{Immunoblotting and immunostaining}

Transfected/transduced HUVECs were harvested and cell lysates were prepared in RIPA buffer (Santa Cruz). Equal amounts of protein were loaded on SDS polyacrylamide gel (Invitrogen) and processed for immunoblotting analysis as previously described $[13,34]$. The protein expression levels of NRP-1 (Santa Cruz Biotechnology \# sc-5541), CD31 (Cell Signaling \# 3528), VE-cadherin (Santa Cruz \# sc-6458), N-cadherin (Abcam \# ab76057), aSMA (Abcam \# ab5694), $\alpha$-actinin (Cell Signaling \# 3134), TGF $\beta 1$ (Abcam \# ab9758), TGFBR1 (Cell Signaling \# 3712), TGFBR2 (Cell Signaling \#11888), SMAD2 (Cell Signaling \# 3122), pSMAD2 (Cell Signaling \# 3101), CTGF (Abcam \# ab6992), Slug (Cell Signaling \# 9585) and GAPDH (Santa Cruz \# sc25778) were measured using specific antibodies. GAPDH was used as a loading control for all immunoblotting experiments. Following incubation with the appropriate horseradish peroxidase-tagged secondary antibodies (Bio-Rad), signals were visualized with an enhanced chemiluminescence detection system for immunoblotting (SuperSignal $^{\mathrm{TM}}$, Life Technologies). Following $24 \mathrm{~h}$ of transfection and $48 \mathrm{~h}$ of transduction, immunofluorescence and immunohistochemistry for NRP-1, CD31, VE-cadherin, $\alpha \mathrm{SMA}, \mathrm{N}$-cadherin and $\alpha$-actinin were performed and visualized using standard immunostaining protocols. Fluorescent microscopy Images were captured using the Zeiss LSM700 confocal microscope and ZEN imaging software was used for image processing.

\section{Capillary-like tube formation assay}

$60 \mu \mathrm{l}$ of Matrigel $^{\mathrm{TM}}$ (Becton Dickinson) was added to each well of the 96-well tissue culture plate (Corning) and allowed to polymerize at $37^{\circ} \mathrm{C}, 5 \% \mathrm{CO}_{2}$ for 45 minutes 
under sterile conditions. $0.5 \times 10^{4}$ transfected/transduced HUVECs were plated onto the surface of the Matrigel ${ }^{\mathrm{TM}}$ and cultured as described earlier. All of the wells were stimulated with VEGF $(50 \mathrm{ng} / \mathrm{ml})$ to induce in vitro tube formation. The capillary-like network formation was observed at regular intervals until $24 \mathrm{~h}$ and photomicrographs were recorded at different time intervals for quantification.

\section{Cell viability assay}

MTT assay of cell viability (Vybrant, Life technologies) was performed to assess growth kinetics according to the manufacturer's protocol. 10,000 BxPC-3 cells transfected with shRNA or scramble minicircle were seeded in each well of 96 well plates $(n=6) .10 \mu 1$ of the $12 \mathrm{mM}$ MTT stock solution was added to each well and incubated at $37^{\circ} \mathrm{C}, 5 \% \mathrm{CO}_{2}$ for 4 hours. $50 \mu \mathrm{l}$ of DMSO (Sigma) was added to each well and mixed thoroughly to solubilize the formazan. The pate was again incubated at $37^{\circ} \mathrm{C}, 5 \% \mathrm{CO}_{2}$ for 10 minutes. Each sample was mixed again and absorbance was read at $540 \mathrm{~nm}$ using an automated plate reader (Molecular Devices).

\section{Human xenograft studies}

Formalin-fixed paraffin-embedded and flash frozen human PDAC tissues were obtained from Dr. David Hedley (OICR, Toronto, Canada). qPCR and immunoblotting for NRP-1, EndMT and fibrosis markers was performed on these samples. Immunohistochemistry, hematoxylin-eosin (H\&E) staining and Masson's trichrome staining (Polybiosciences Inc.) was performed on the human PDAC tissue following the manufacturer's guidelines. NRP-1 levels and fibrosis were quantified with light microscopy using the ImageJ software (NIH).

\section{Generation of orthotopic tumor model in athymic rats}

The in vivo study protocol was approved by the Animal Care and Use Committee at the Keenan Research Centre for Biomedical Science, Li Ka Shing Knowledge Institute, St Michael's Hospital, University of Toronto. Rowett Nude (RNU, strain 316) rats were purchased from Charles River. BxPC-3 human pancreatic cancer cells were purchased from ATCC and cultured in RPMI 1640 medium (ATCC) supplemented with 10\% fetal bovine serum and antibiotics. $\left(5 \times 10^{6}\right)$ cells were injected $(200 \mu \mathrm{l}$ cells in RPMI medium $+200 \mu$ Matrigel $^{\mathrm{TM}}$ ) in the flank of athymic rats to establish heterotopic tumors. Pancreatic tumors grown subcutaneously were then harvested at exponential growth phase under aseptic conditions to establish the orthotopic model. Viable tissues were cut and minced into $3-\mathrm{mm}^{3}$ pieces. In recipient RNU rats, the tail of the pancreas, located in the splenorenal ligament within the spleen, was gently exteriorized via laparotomy. Five tumor fragments were sutured with $7-0$ surgical sutures onto the pancreas so that tumor tissue is completely surrounded by pancreatic parenchyma. The pancreas was placed back in the original position and the muscle and skin were sutured. Post-mortem, tumor tissues were stained with H\&E staining and Masson's trichrome staining for characterization.

\section{Contrast enhanced ultrasound perfusion imaging and ultrasound-mediated loss of NRP-1 in vivo}

The contrast-enhanced ultrasound (CEU) perfusion and gene delivery studies were performed as previously described [80, 81]. CEU perfusion imaging of tumors was performed at 4 and 8 weeks after implantation surgery. At 4 weeks post tumor implantation surgery, ultrasoundtargeted microbubble destruction (UTMD) [80-82] was performed for NRP-1 silencing. For UTMD, shNRP-1 minicircle (SBI Biosciences) DNA vector $(200 \mu \mathrm{g})$ was charge coupled to cationic microbubbles $\left(1 \times 10^{9}\right)$ and the suspension was administered intravenously during the external application of high power ultrasound over the pancreas $(n=4)$. Scramble minicircle (SBI Biosciences) DNA vector $(200 \mu \mathrm{g})$ was delivered by UTMD as an appropriate control in a separate group of animals $(n=4)$. Animals were sacrificed 4 weeks after gene delivery ( 8 weeks post implantation). Tumor tissue and remote organs were collected for evaluation of tumor volume and other downstream analyses as previously described.

\section{Statistical analysis}

All data were expressed as mean \pm SD unless otherwise indicated. The Student's $t$-test was applied when the means of two groups were being compared. Differences between multiple means were evaluated by One-way ANOVA (GraphPad Prism5) followed by Tukey's posthoc test to compare individual means. $P$ value of $<0.05$ was considered to be statistically significant. Spearman's Rho test was applied to perform bivariate correlation analysis between two variables, where values of $\mathrm{R}$ closer to 1 indicated positive correlation.

\section{ACKNOWLEDGMENTS AND GRANT SUPPORT}

This work was supported by an Equipment Grant from the Canadian Foundation for Innovation, Ottawa, Ontario, Canada and a grant from the Krembil Foundation. PNM is a recipient of the Peterborough K.M. Hunter Charitable Foundation Graduate Award, Faculty of Medicine, University of Toronto. HL-P is supported by the Brazilian Ball Chair in Cardiovascular Research from St. Michael's Hospital, University of Toronto, and by an Early Researcher Award from the Ministry of Research and Innovation, Ontario, Canada. 


\section{CONFLICTS OF INTEREST}

The authors declare no conflicts of interest.

\section{REFERENCES}

1. Siegel RL, Miller KD, Jemal A. Cancer statistics, 2015. CA Cancer J Clin. 2015; 65:5-29.

2. Jemal A, Center MM, DeSantis C, Ward EM. Global patterns of cancer incidence and mortality rates and trends. Cancer Epidemiol Biomarkers Prev. 2010; 19:1893-1907.

3. Weir HK, Thompson TD, Soman A, Moller B, Leadbetter S. The past, present, and future of cancer incidence in the United States: 1975 through 2020. Cancer. 2015; 121:1827-1837.

4. Yadav D, Lowenfels AB. The epidemiology of pancreatitis and pancreatic cancer. Gastroenterology. 2013; 144:1252-1261.

5. Malvezzi M, Bertuccio P, Levi F, La Vecchia C, Negri E. European cancer mortality predictions for the year 2014 . Ann Oncol. 2014; 25:1650-1656.

6. Beger HG, Rau B, Gansauge F, Leder G, Schwarz M, Poch B. Pancreatic cancer-low survival rates. Dtsch Arztebl Int. 2008; 105:255-262.

7. Akinleye A, Iragavarapu C, Furqan M, Cang S, Liu D. Novel agents for advanced pancreatic cancer. Oncotarget. 2015; 6:39521-39537. doi: 10.18632/oncotarget.3999.

8. Ferrara N, Kerbel RS. Angiogenesis as a therapeutic target. Nature. 2005; 438:967-974.

9. Kerbel R, Folkman J. Clinical translation of angiogenesis inhibitors. Nat Rev Cancer. 2002; 2:727-739.

10. Jain RK. Lessons from multidisciplinary translational trials on anti-angiogenic therapy of cancer. Nat Rev Cancer. 2008; 8:309-316.

11. Heath VL, Bicknell R. Anticancer strategies involving the vasculature. Nat Rev Clin Oncol. 2009; 6:395-404.

12. Kindler HL, Niedzwiecki D, Hollis D, Sutherland S, Schrag D, Hurwitz H, Innocenti F, Mulcahy MF, O’Reilly E, Wozniak TF, Picus J, Bhargava P, Mayer RJ, et al. Gemcitabine plus bevacizumab compared with gemcitabine plus placebo in patients with advanced pancreatic cancer: phase III trial of the Cancer and Leukemia Group B (CALGB 80303). J Clin Oncol. 2010; 28:3617-3622.

13. Olive KP, Jacobetz MA, Davidson CJ, Gopinathan A, McIntyre D, Honess D, Madhu B, Goldgraben MA, Caldwell ME, Allard D, Frese KK, Denicola G, Feig C, et al. Inhibition of Hedgehog signaling enhances delivery of chemotherapy in a mouse model of pancreatic cancer. Science. 2009; 324:1457-1461.

14. Hernandez-Munoz I, Skoudy A, Real FX, Navarro P. Pancreatic ductal adenocarcinoma: cellular origin, signaling pathways and stroma contribution. Pancreatology. 2008; 8:462-469.

15. Erkan M. Understanding the stroma of pancreatic cancer: co-evolution of the microenvironment with epithelial carcinogenesis. J Pathol. 2013; 231:4-7.
16. Hartel M, Di Mola FF, Gardini A, Zimmermann A, Di Sebastiano P, Guweidhi A, Innocenti P, Giese T, Giese N, Buchler MW, Friess H. Desmoplastic reaction influences pancreatic cancer growth behavior. World J Surg. 2004; 28:818-825.

17. Kadaba R, Birke H, Wang J, Hooper S, Andl CD, Di Maggio F, Soylu E, Ghallab M, Bor D, Froeling FE, Bhattacharya S, Rustgi AK, Sahai E, et al. Imbalance of desmoplastic stromal cell numbers drives aggressive cancer processes. J Pathol. 2013; 230:107-117.

18. Whatcott CJ, Diep CH, Jiang P, Watanabe A, LoBello J, Sima C, Hostetter G, Shepard HM, Von Hoff DD, Han H. Desmoplasia in Primary Tumors and Metastatic Lesions of Pancreatic Cancer. Clin Cancer Res. 2015; 21:3561-3568.

19. Sugimoto H, Mundel TM, Kieran MW, Kalluri R. Identification of fibroblast heterogeneity in the tumor microenvironment. Cancer Biol Ther. 2006; 5:1640-1646.

20. Kalluri R, Zeisberg M. Fibroblasts in cancer. Nat Rev Cancer. 2006; 6:392-401.

21. Tlsty TD, Hein PW. Know thy neighbor: stromal cells can contribute oncogenic signals. Curr Opin Genet Dev. 2001; 11:54-59.

22. Bierie B, Moses HL. TGF-beta and cancer. Cytokine Growth Factor Rev. 2006; 17:29-40.

23. Neesse A, Michl P, Frese KK, Feig C, Cook N, Jacobetz MA, Lolkema MP, Buchholz M, Olive KP, Gress TM, Tuveson DA. Stromal biology and therapy in pancreatic cancer. Gut. 2011; 60:861-868.

24. Jacobetz MA, Chan DS, Neesse A, Bapiro TE, Cook N, Frese KK, Feig C, Nakagawa T, Caldwell ME, Zecchini HI, Lolkema MP, Jiang P, Kultti A, et al. Hyaluronan impairs vascular function and drug delivery in a mouse model of pancreatic cancer. Gut. 2013; 62:112-120.

25. Schober M, Jesenofsky R, Faissner R, Weidenauer C, Hagmann W, Michl P, Heuchel RL, Haas SL, Lohr JM. Desmoplasia and chemoresistance in pancreatic cancer. Cancers. 2014; 6:2137-2154.

26. Lohr JM, Haas SL, Bechstein WO, Bodoky G, Cwiertka K, Fischbach W, Folsch UR, Jager D, Osinsky D, Prausova J, Schmidt WE, Lutz MP. Cationic liposomal paclitaxel plus gemcitabine or gemcitabine alone in patients with advanced pancreatic cancer: a randomized controlled phase II trial. Ann Oncol. 2012; 23:1214-1222.

27. Von Hoff DD, Ramanathan RK, Borad MJ, Laheru DA, Smith LS, Wood TE, Korn RL, Desai N, Trieu V, Iglesias JL, Zhang $\mathrm{H}$, Soon-Shiong $\mathrm{P}$, Shi $\mathrm{T}$, et al. Gemcitabine plus nab-paclitaxel is an active regimen in patients with advanced pancreatic cancer: a phase I/II trial. J Clin Oncol. 2011; 29:4548-4554.

28. Khan S, Ebeling MC, Chauhan N, Thompson PA, Gara RK, Ganju A, Yallapu MM, Behrman SW, Zhao H, Zafar N, Singh MM, Jaggi M, Chauhan SC. Ormeloxifene suppresses desmoplasia and enhances sensitivity of gemcitabine in pancreatic cancer. Cancer Res. 2015; 75:2292-2304. 
29. Zeisberg EM, Tarnavski O, Zeisberg M, Dorfman AL, McMullen JR, Gustafsson E, Chandraker A, Yuan X, $\mathrm{Pu}$ WT, Roberts AB, Neilson EG, Sayegh MH, Izumo S, et al. Endothelial-to-mesenchymal transition contributes to cardiac fibrosis. Nat Med. 2007; 13:952-961.

30. Li J, Qu X, Bertram JF. Endothelial-myofibroblast transition contributes to the early development of diabetic renal interstitial fibrosis in streptozotocin-induced diabetic mice. Am J Pathol. 2009; 175:1380-1388.

31. Piera-Velazquez S, Li Z, Jimenez SA. Role of endothelialmesenchymal transition (EndoMT) in the pathogenesis of fibrotic disorders. Am J Pathol. 2011; 179:1074-1080.

32. Markwald RR, Fitzharris TP, Smith WN. Sturctural analysis of endocardial cytodifferentiation. Dev Biol. 1975; 42:160-180.

33. Lee JG, Kay EP. FGF-2-mediated signal transduction during endothelial mesenchymal transformation in corneal endothelial cells. Exp Eye Res. 2006; 83:1309-1316.

34. Potenta S, Zeisberg E, Kalluri R. The role of endothelial-tomesenchymal transition in cancer progression. Br J Cancer. 2008; 99:1375-1379.

35. Zeisberg EM, Potenta S, Xie L, Zeisberg M, Kalluri R. Discovery of endothelial to mesenchymal transition as a source for carcinoma-associated fibroblasts. Cancer Res. 2007; 67:10123-10128.

36. Medici D, Potenta S, Kalluri R. Transforming growth factor-beta2 promotes Snail-mediated endothelialmesenchymal transition through convergence of Smaddependent and Smad-independent signalling. Biochem J. 2011; 437:515-520.

37. Rosenbloom J, Castro SV, Jimenez SA. Narrative review: fibrotic diseases: cellular and molecular mechanisms and novel therapies. Ann Intern Med. 2010; 152:159-166.

38. Medici D, Shore EM, Lounev VY, Kaplan FS, Kalluri R, Olsen BR. Conversion of vascular endothelial cells into multipotent stem-like cells. Nat Med. 2010; 16:1400-1406.

39. Singh KK, Lovren F, Pan Y, Quan A, Ramadan A, Matkar PN, Ehsan M, Sandhu P, Mantella LE, Gupta N, Teoh H, Parotto M, Tabuchi A, et al. The essential autophagy gene ATG7 modulates organ fibrosis via regulation of endothelial-to-mesenchymal transition. J Biol Chem. 2015; 290:2547-2559.

40. He Z, Tessier-Lavigne M. Neuropilin is a receptor for the axonal chemorepellent Semaphorin III. Cell. 1997; 90:739-751.

41. Guttmann-Raviv N, Kessler O, Shraga-Heled N, Lange T, Herzog Y, Neufeld G. The neuropilins and their role in tumorigenesis and tumor progression. Cancer Lett. 2006; 231:1-11.

42. Bielenberg DR, Pettaway CA, Takashima S, Klagsbrun M. Neuropilins in neoplasms: expression, regulation, and function. Exp Cell Res. 2006; 312:584-593.

43. Prud'homme GJ, Glinka Y. Neuropilins are multifunctional coreceptors involved in tumor initiation, growth, metastasis and immunity. Oncotarget. 2012; 3:921-939. doi: 10.18632/ oncotarget.626.
44. Matsushita A, Gotze T, Korc M. Hepatocyte growth factormediated cell invasion in pancreatic cancer cells is dependent on neuropilin-1. Cancer Res. 2007; 67:10309-10316.

45. Fukasawa M, Matsushita A, Korc M. Neuropilin-1 interacts with integrin betal and modulates pancreatic cancer cell growth, survival and invasion. Cancer Biol Ther. 2007; 6:1173-1180.

46. Evans IM, Yamaji M, Britton G, Pellet-Many C, Lockie C, Zachary IC, Frankel P. Neuropilin-1 signaling through p130Cas tyrosine phosphorylation is essential for growth factor-dependent migration of glioma and endothelial cells. Mol Cell Biol. 2011; 31:1174-1185.

47. Jia H, Cheng L, Tickner M, Bagherzadeh A, Selwood D, Zachary I. Neuropilin-1 antagonism in human carcinoma cells inhibits migration and enhances chemosensitivity. Br J Cancer. 2010; 102:541-552.

48. Beck B, Driessens G, Goossens S, Youssef KK, Kuchnio A, Caauwe A, Sotiropoulou PA, Loges S, Lapouge G, Candi A, Mascre G, Drogat B, Dekoninck S, et al. A vascular niche and a VEGF-Nrp1 loop regulate the initiation and stemness of skin tumours. Nature. 2011; 478:399-403.

49. Fuh G, Garcia KC and de Vos AM. The interaction of neuropilin-1 with vascular endothelial growth factor and its receptor flt-1. J Biol Chem. 2000; 275:26690-26695.

50. West DC, Rees CG, Duchesne L, Patey SJ, Terry CJ, Turnbull JE, Delehedde M, Heegaard CW, Allain F, Vanpouille C, Ron D, Fernig DG. Interactions of multiple heparin binding growth factors with neuropilin-1 and potentiation of the activity of fibroblast growth factor-2. J Biol Chem. 2005; 280:13457-13464.

51. Glinka Y, Stoilova S, Mohammed N, Prud'homme GJ. Neuropilin-1 exerts co-receptor function for TGF-beta-1 on the membrane of cancer cells and enhances responses to both latent and active TGF-beta. Carcinogenesis. 2011; 32:613-621.

52. Migdal M, Huppertz B, Tessler S, Comforti A, Shibuya M, Reich R, Baumann H, Neufeld G. Neuropilin-1 is a placenta growth factor-2 receptor. J Biol Chem. 1998; 273:22272-22278.

53. Soker S, Takashima S, Miao HQ, Neufeld G, Klagsbrun M. Neuropilin-1 is expressed by endothelial and tumor cells as an isoform-specific receptor for vascular endothelial growth factor. Cell. 1998; 92:735-745.

54. Guo HF, Vander Kooi CW. Neuropilin Functions as an Essential Cell Surface Receptor. J Biol Chem. 2015; 290:29120-29126.

55. Di Guglielmo GM, Le Roy C, Goodfellow AF, Wrana JL. Distinct endocytic pathways regulate TGF-beta receptor signalling and turnover. Nat Cell Biol. 2003; 5:410-421.

56. Fuxe J, Vincent T, Garcia de Herreros A. Transcriptional crosstalk between TGF-beta and stem cell pathways in tumor cell invasion: role of EMT promoting Smad complexes. Cell Cycle. 2010; 9:2363-2374.

57. Cesi V, Casciati A, Sesti F, Tanno B, Calabretta B, Raschella G. TGFbeta-induced c-Myb affects the expression of EMT-associated genes and promotes invasion of ER+ breast cancer cells. Cell Cycle. 2011; 10:4149-4161. 
58. Tian M, Neil JR, Schiemann WP. Transforming growth factor-beta and the hallmarks of cancer. Cell Signal. 2011; 23:951-962.

59. Wendt MK, Smith JA, Schiemann WP. p130Cas is required for mammary tumor growth and transforming growth factor-beta-mediated metastasis through regulation of Smad2/3 activity. J Biol Chem. 2009; 284:34145-34156.

60. Fukahi K, Fukasawa M, Neufeld G, Itakura J, Korc M. Aberrant expression of neuropilin-1 and -2 in human pancreatic cancer cells. Clin Cancer Res. 2004; 10:581-590.

61. Wey JS, Gray MJ, Fan F, Belcheva A, McCarty MF, Stoeltzing O, Somcio R, Liu W, Evans DB, Klagsbrun M, Gallick GE, Ellis LM. Overexpression of neuropilin-1 promotes constitutive MAPK signalling and chemoresistance in pancreatic cancer cells. Br J Cancer. 2005; 93:233-241.

62. Hansel DE, Wilentz RE, Yeo CJ, Schulick RD, Montgomery E, Maitra A. Expression of neuropilin-1 in high-grade dysplasia, invasive cancer, and metastases of the human gastrointestinal tract. Am J Surg Pathol. 2004; 28:347-356.

63. Chu W, Song X, Yang X, Ma L, Zhu J, He M, Wang Z, $\mathrm{Wu}$ Y. Neuropilin-1 promotes epithelial-to-mesenchymal transition by stimulating nuclear factor-kappa B and is associated with poor prognosis in human oral squamous cell carcinoma. PloS one. 2014; 9:e101931.

64. Adham SA, Al Harrasi I, Al Haddabi I, Al Rashdi A, Al Sinawi S, Al Maniri A, Ba-Omar T, Coomber BL. Immunohistological insight into the correlation between neuropilin-1 and epithelial-mesenchymal transition markers in epithelial ovarian cancer. J Histochem Cytochem. 2014; 62:619-631.

65. Yoshimatsu Y, Watabe T. Roles of TGF-beta signals in endothelial-mesenchymal transition during cardiac fibrosis. Int J Inflam. 2011; 2011:724080.

66. Zhao L, Lin L, Pan C, Shi M, Liao Y, Bin J, Liao W. Flotillin-2 promotes nasopharyngeal carcinoma metastasis and is necessary for the epithelial-mesenchymal transition induced by transforming growth factor-beta. Oncotarget. 2015; 6:9781-9793. doi: 10.18632/oncotarget.3382.

67. Truty MJ, Urrutia R. Basics of TGF-beta and pancreatic cancer. Pancreatology. 2007; 7:423-435.

68. Neuzillet C, de Gramont A, Tijeras-Raballand A, de Mestier L, Cros J, Faivre S, Raymond E. Perspectives of TGF-beta inhibition in pancreatic and hepatocellular carcinomas. Oncotarget. 2014; 5:78-94. doi: 10.18632/ oncotarget.1569.

69. Belcik JT, Davidson BP, Foster T, Qi Y, Zhao Y, Peters D, Lindner JR. Contrast-enhanced ultrasound assessment of impaired adipose tissue and muscle perfusion in insulinresistant mice. Circ Cardiovasc Imaging. 2015; 8.

70. Huang M, Nguyen P, Jia F, Hu S, Gong Y, de Almeida PE, Wang L, Nag D, Kay MA, Giaccia AJ, Robbins RC, Wu JC. Double knockdown of prolyl hydroxylase and factor-inhibiting hypoxia-inducible factor with nonviral minicircle gene therapy enhances stem cell mobilization and angiogenesis after myocardial infarction. Circulation. 2011; 124:S46-54.
71. Lijkwan MA, Hellingman AA, Bos EJ, van der Bogt KE, Huang M, Kooreman NG, de Vries MR, Peters HA, Robbins RC, Hamming JF, Quax PH, Wu JC. Short hairpin RNA gene silencing of prolyl hydroxylase-2 with a minicircle vector improves neovascularization of hindlimb ischemia. Hum Gene Ther. 2014; 25:41-49.

72. Aspalter IM, Gordon E, Dubrac A, Ragab A, Narloch J, Vizan P, Geudens I, Collins RT, Franco CA, Abrahams CL, Thurston G, Fruttiger M, Rosewell I, et al. Alk1 and Alk5 inhibition by Nrp1 controls vascular sprouting downstream of Notch. Nat Commun. 2015; 6:7264.

73. Hirota S, Clements TP, Tang LK, Morales JE, Lee HS, Oh SP, Rivera GM, Wagner DS, McCarty JH. Neuropilin 1 balances beta 8 integrin-activated TGFbeta signaling to control sprouting angiogenesis in the brain. Development. $2015 ; 142: 4363-4373$.

74. Goumans MJ, Valdimarsdottir G, Itoh S, Lebrin F, Larsson J, Mummery C, Karlsson S and ten Dijke P. Activin receptorlike kinase (ALK)1 is an antagonistic mediator of lateral TGFbeta/ALK5 signaling. Mol Cell. 2003; 12:817-828.

75. Zhang YE. Non-Smad pathways in TGF-beta signaling. Cell Res. 2009; 19:128-139.

76. Nasarre P, Gemmill RM, Potiron VA, Roche J, Lu X, Baron AE, Korch C, Garrett-Mayer E, Lagana A, Howe PH, Drabkin HA. Neuropilin-2 Is upregulated in lung cancer cells during TGF-beta1-induced epithelial-mesenchymal transition. Cancer Res. 2013; 73:7111-7121.

77. Deer EL, Gonzalez-Hernandez J, Coursen JD, Shea JE, Ngatia J, Scaife CL, Firpo MA, Mulvihill SJ. Phenotype and genotype of pancreatic cancer cell lines. Pancreas. 2010; 39:425-435.

78. Metildi CA, Kaushal S, Hoffman RM, Bouvet M. In vivo serial selection of human pancreatic cancer cells in orthotopic mouse models produces high metastatic variants irrespective of Kras status. J Surg Res. 2013; 184:290-298.

79. Huynh AS, Abrahams DF, Torres MS, Baldwin MK, Gillies RJ, Morse DL. Development of an orthotopic human pancreatic cancer xenograft model using ultrasound guided injection of cells. PloS one. 2011; 6:e20330.

80. Cao WJ, Rosenblat JD, Roth NC, Kuliszewski MA, Matkar PN, Rudenko D, Liao C, Lee PJ, Leong-Poi H. Therapeutic Angiogenesis by Ultrasound-Mediated MicroRNA-126-3p Delivery. Arterioscler Thromb Vasc Biol. 2015; 35:2401-2411.

81. Fujii H, Matkar P, Liao C, Rudenko D, Lee PJ, Kuliszewski MA, Prud'homme GJ, Leong-Poi H. Optimization of Ultrasound-mediated Anti-angiogenic Cancer Gene Therapy. Mol Ther Nucleic Acids. 2013; 2:e94.

82. Lee PJ, Rudenko D, Kuliszewski MA, Liao C, Kabir MG, Connelly KA, Leong-Poi H. Survivin gene therapy attenuates left ventricular systolic dysfunction in doxorubicin cardiomyopathy by reducing apoptosis and fibrosis. Cardiovasc Res. 2014; 101:423-433.

83. Yue B, Ma JF, Yao G, Yang MD, Cheng H, Liu GY. Knockdown of neuropilin-1 suppresses invasion, angiogenesis, 
and increases the chemosensitivity to doxorubicin in osteosarcoma cells-an in vitro study. Eur Rev Med Pharmacol Sci. 2014; 18:1735-1741.

84. Zheng X, Carstens JL, Kim J, Scheible M, Kaye J, Sugimoto H, Wu CC, LeBleu VS, Kalluri R. Epithelial-tomesenchymal transition is dispensable for metastasis but induces chemoresistance in pancreatic cancer. Nature. 2015; 527:525-530.
85. Lohse I, Borgida A, Cao P, Cheung M, Pintilie M, Bianco T, Holter S, Ibrahimov E, Kumareswaran R, Bristow RG, Tsao MS, Gallinger S, Hedley DW. BRCA1 and BRCA2 mutations sensitize to chemotherapy in patient-derived pancreatic cancer xenografts. Br J Cancer. 2015; 113:425-432. 